\title{
Change detection on a hunch: Pre-attentive vision allows "sensing" of unique feature changes
}

\author{
Felix Ball $^{1,2} \cdot$ Niko A. Busch ${ }^{1,2}$
}

Published online: 9 September 2015

(C) The Psychonomic Society, Inc. 2015

\begin{abstract}
Studies on change detection and change blindness have investigated the nature of visual representations by testing the conditions under which observers are able to detect when an object in a complex scene changes from one moment to the next. Several authors have proposed that change detection can occur without identification of the changing object, but the perceptual processes underlying this phenomenon are currently unknown. We hypothesized that change detection without localization or identification occurs when the change happens outside the focus of attention. Such changes would usually go entirely unnoticed, unless the change brings about a modification of one of the feature maps representing the scene. Thus, the appearance or disappearance of a unique feature might be registered even in the absence of focused attention and without feature binding, allowing for change detection, but not localization or identification. We tested this hypothesis in three experiments, in which changes either involved colors that were already present elsewhere in the display or entirely unique colors. Observers detected whether any change had occurred and then localized or identified the change. Change detection without localization occurred
\end{abstract}

Electronic supplementary material The online version of this article (doi:10.3758/s13414-015-0963-9) contains supplementary material, which is available to authorized users.

Niko A. Busch

niko.busch@charite.de

1 Institute of Medical Psychology, Charité University Medicine, Luisenstraße 57, 10117 Berlin, Germany

2 Berlin School of Mind and Brain, Humboldt-University, Berlin, Germany almost exclusively when changes involved a unique color. Moreover, change detection without localization for unique feature changes was independent of the number of objects in the display and independent of change identification. These findings suggest that pre-attentive registration of a change on a feature map can give rise to a conscious experience even when feature binding has failed: that something has changed without knowing what or where.

Keywords Change blindness · Visual awareness - Visual working memory
Abbreviations
$\mathrm{Det}^{+} \mathrm{Loc}^{+}$
Change detection with localization
$\mathrm{Det}^{+} \mathrm{Loc}^{-}$
Change detection without localization
$\operatorname{Det}^{-} \mathrm{Loc}^{+}$ (also referred to as "sensing")
Det $^{-}$Loc $^{-}$ Change localization without detection
$\mathrm{Det}^{+} \mathrm{Loc}^{-} \mathrm{Id}^{+}$ Change blindness
$\operatorname{Det}^{+} \operatorname{Loc}^{-} I^{-}$
Change detection without localization but with identification
Det- $d^{\prime}$
Change detection without localization and without identification
Loc-d
Detection-d'
Sens-d'
Localization-d'
$N F C$
Sensing-d'
$U F C$
Non-unique feature change
Unique feature change

\section{Introduction}

The ultimate goal for research on visual cognition has been to establish how visual input from the outside world is represented in the visual system, and what types of behavior and phenomenological experience these representations 
support. Phenomenology suggests that we have access to a detailed and stable representation of a visual scene. However, this intuition has been challenged by results obtained with the "change blindness" paradigm (see Rensink, 2002; Simons \& Rensink, 2005, for reviews). This line of research has demonstrated that observers are surprisingly poor at detecting substantial changes in a scene if the change occurs simultaneously with a brief visual disruption, be it a saccade (McConkie \& Currie, 1996; Hayhoe et al., 1998), an eye blink (O'Regan et al., 2000), a flicker (Ball et al., 2013; Rensink et al., 1997), or a distracting stimulus (O'Regan et al., 1999). Under these conditions, change detection cannot be based on detecting a motion or contrast transient, but depends on encoding, preserving, and comparing object representations of pre-change and post-change objects. Thus, the change blindness phenomenon points to a limit in our ability to represent, process, and maintain visual scenes.

Studies of change blindness often assume that detecting a change (knowing that a change has occurred) also allows for localizing and identifying the changing object (seeing what has changed). However, a number of studies have compared behavioral performance in detection and identification tasks and found that observers may correctly detect the presence of a change without being able to identify what has changed (Agostinelli et al., 1986; Busch et al., 2010b; Becker et al., 2000; Turatto \& Bridgeman, 2005; Hughes et al., 2012). Moreover, Rensink (2004) studied the phenomenological experience that accompanies visual changes and found that observers often reported that "something is changing" before they were able to report the location and identity of the changing object. Rensink termed this phenomenon of perceiving a change without an accompanying visual experience "sensing", as opposed to "seeing" the object's identity and location, and concluded that "sensing" and "seeing" are not only phenomenologically distinct, but are also associated with distinct perceptual processes.

The ensuing question is what visual information enables "seeing" and "sensing", or detection with and without localization/identification, respectively. Galpin et al. (2008) and Busch et al. (2010b) have suggested that sensing the presence of a change without identification of the changing object occurs when features of the changing object are registered pre-attentively, but the subsequent stage of feature integration fails. Most variants of feature integration theories conceptualize early vision as a multi-stage process (see Quinlan, 2003, for an overview). At the first stage, visual input is decomposed into basic feature dimensions such as color, orientation, or texture. Features are thought to be represented on specialized feature maps that code only the presence of a feature (e.g., presence of the color red or vertical line orientation), but not the feature's location or to which object it belongs. Up to this point, processing is thought to be largely pre-attentive and efficient, and features are considered to be "free-floating" or "unbound". According to Treisman and Gelade (1980) and Treisman and Gormican (1988), pre-attentive registration of features can allow for the detection of a feature's presence, but not for its localization or for recognition of a unified object. Thus, in visual search tasks, the time required to find a target that is defined by a unique feature in the display is largely independent of the number of items in the display (set size) since pre-attentive registration of feature-presence on a feature map is sufficient for detection of feature presence. At the next stage, spatial attention acts as a "glue" that binds together features and their locations to form coherent object representations, and makes these objects available to conscious perception. This integration stage is thought to be inefficient, attention-dependent, and capacity-limited. Consequently, the speed of visual search for targets that are defined by feature combinations is strongly dependent on set size.

Accordingly, feature integration theory suggests that the feeling of sensing and change detection without localization or identification is based on the following chain of events: when a complex visual scene is presented, objects in this scene are represented pre-attentively on multiple feature maps, each carrying a different dimension (color, orientation, etc.). Only a small subset of objects within the current focus of spatial attention is bound into coherent object representations, and conscious identification of a change is restricted to changes within this area. When a change occurs outside the focus of attention, this change will go undetected and the observer will be change blind, unless a new, unique feature gets represented on a feature map or conversely, disappears from the map. Such pre-attentive registration of this feature change would enable the observer to report that something has changed, but not to localize or identify the changing object. In line with this suggestion, Busch et al. (2010a) and Busch et al. (2010b) demonstrated that change detection without localization or identification (as compared to actual change blindness) is associated with the so-called selection negativity, an electrophysiological correlate of feature-selective attention. By contrast, only change localization and identification were additionally accompanied by an index of a shift of spatial attention towards the changing object (the N2pc component). Moreover, Rensink (2004) demonstrated that presenting a colored blank field, rather than a gray blank field, in between scenes considerably delayed the onset of sensing specifically for color changes. By contrast, the colored blank screen had only a moderate effect on seeing for all types of changes. In line with our proposal, this finding may indicate that the colored blank field interfered with sensing by "filling" the feature map with the relevant color such that the color change could not be registered by detecting a change on this feature map. 
In the present study, we tested key predictions derived from this hypothesis. Specifically, we predicted different patterns of performance for changes of unique features (here colors), which occur only once in the display and non-unique features, which are present in multiple objects:

1. If detection without localization/identification is based on pre-attentive feature registration of unique feature changes, the frequency of detection without localization of unique feature changes should be set sizeindependent (Experiment 1);

2. Observers should be able to detect, localize, and identify changes in unique as well as non-unique features. By contrast, correct change detection without localization/identification should be restricted to unique feature changes (Experiments 1, 2 and 3).

To this end, we presented scenes with multiple objects, each composed of multi-colored line-elements. In each object, one element was filled in with a unique color that appeared nowhere else in the display, while the remaining elements contained colors which recurred (non-unique) on the display. Changes consisted of either the appearance or disappearance of such a unique color (unique feature change), or the replacement of one non-unique color with another non-unique color (non-unique feature change). In Experiment 1, set size was manipulated in a "one-shot" change detection paradigm, in which only a single change occurs on each trial. Observers were required to detect the presence or absence of a change and to localize where the change had occurred. In Experiment 2, set size was kept constant and observers were to detect, localize and identify the changes. To conform with previous studies (Rensink, 2004; Rensink et al., 1997; Simons et al., 2005), Experiment 3 used a flicker paradigm, in which original and modified scene alternated several times.

\section{Experiment 1: Set size dependence of change detection without localization}

\section{Methods}

\section{Participants}

A total of 24 participants (mean age: $23.4 \pm 3.7$ SD; 16 women, eight men; two left-handed) were tested after giving signed informed consent. All participants reported to be free of neurological or psychiatric disorders and had normal or corrected-to-normal visual acuity. The experimental protocol was approved by the ethics committee of the German Psychological Society (DGPS).

\section{Apparatus and stimuli}

The experiment was written in Matlab (Mathworks Inc.) using the Psychophysics Toolbox (Brainard, 1997). Participants were seated in a dark, sound-attenuated chamber. Stimuli were presented on a calibrated 19-inch CRT monitor with a resolution of $1280 \times 1024$ and refresh rate of $100 \mathrm{~Hz}$, placed at a distance of $56 \mathrm{~cm}$ from the participants' eyes. Head position was stabilized using a chin-rest.

Scenes consisted of three, five, or seven objects, in counterbalanced order. Objects subtended $1.26^{\circ}$ visual angle, and each object was presented at a distance of $1.5^{\circ}$ or $3.5^{\circ}$ from the central fixation cross. Each object consisted of two parallel colored bars surrounded by a black square (see Fig. 1 for an example). One bar of each object was colored with a unique color that appeared only once in the scene. The second bar of each object was filled in with one of two non-unique colors that appeared repeatedly in the scene and were randomly distributed across all objects. We used a set of ten colors, comprised of five different hues (yellow, red, green, blue and purple) and two levels of brightness of each hue.

\section{Procedure}

The experiment consisted of 480 trials plus 24 training trials. On each trial, an original scene (A) and a changed scene $\left(\mathrm{A}^{\prime}\right)$ were presented in a sequence of three presentations of $500 \mathrm{~ms}$ each, and a change occurred either between the first two presentations $\left(\mathrm{A}-\mathrm{A}^{\prime}-\mathrm{A}^{\prime}\right)$ or between the last two presentations ( $\left.\mathrm{A}-\mathrm{A}-\mathrm{A}^{\prime}\right)$. Blank displays (white) of 100 ms duration between scenes served to disrupt transient change signals. Two change types were used: a change could either involve the substitution of a unique color (referred to "unique feature change") or a non-unique color (referred to "non-unique feature change") within a single object. Importantly, changes were always made within one hue (pale to dark color or vice versa). Thus, we only changed e.g., a pale red bar into a dark red bar or e.g., a dark blue bar into a pale blue bar. The luminance difference between pale and dark bars was equal across different hues. Across different trials, the same colors were used for the unique feature (e.g., dark blue bar or pale blue bar was unique in the scene) and non-unique feature changes (e.g., dark blue and pale blue bars recurred in the scene). After the presentation sequence, participants answered two questions. In the detection task, participants reported when the change had occurred, i.e., either between presentations 1 and 2 or between 2 and 3. For the localization task, the locations of all objects were highlighted with placeholders, and participants reported the location were the change had occurred by clicking on the appropriate location. Blank displays were presented during the inter-trial intervals 
(500-1500 ms). Participants were instructed to maintain central fixation during stimulus presentation and to answer as accurately as possible (Fig. 1).

\section{Analysis}

The analysis was designed to test how performance in the change detection and localization task depended on

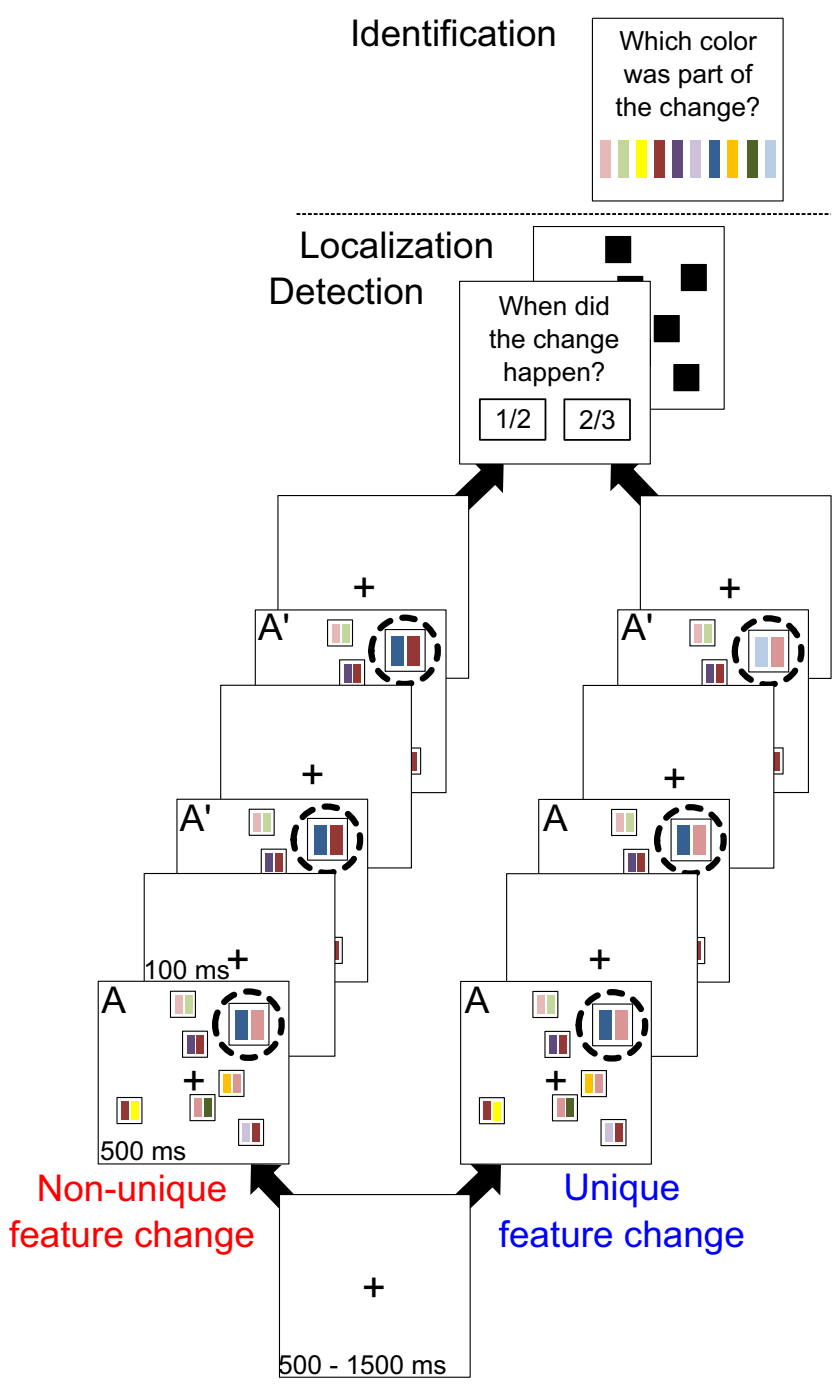

Fig. 1 Experimental Design Experiment 1: Each trial was preceded by a blank display (500-1500 ms). The original scene (A) was substituted by the modified scene $\left(\mathrm{A}^{\prime}\right)$ within a sequence of three displays (500 ms each) interrupted by short blank screens (100 ms). Changes occurred either after the first display $\left(\mathrm{A}-\mathrm{A}^{\prime}-\mathrm{A}^{\prime}\right.$ sequence, exemplarily illustrated for non-unique feature change) or after the second display $\left(\mathrm{A}-\mathrm{A}-\mathrm{A}^{\prime}\right.$ sequence, exemplarily illustrated for unique feature change). Unique and non-unique feature changes were presented in either sequence. The target object is magnified and marked with a dotted circle for illustration. Change displays were followed only by a detection task and localization task display. Experiment 2: Same as Experiment 1 but this time change displays were followed by a detection task, localization task, and identification task display the type of change (unique feature vs. non-unique feature change) and how the type of change interacted with set size. Specifically, we tested the hypothesis that change detection without localization is set size-independent for unique, but not for non-unique feature changes. In the remainder of the manuscript, we denote the behavioral outcome in the change detection and localization tasks with "Det" and "Loc", respectively. Correct and incorrect responses are denoted as + and - , respectively. For example, correct change detection without localization is denoted as Det $^{+}$Loc $^{-}$. Furthermore, we will use abbreviations to label unique feature change (UFC) and non-unique feature change (NFC). $\mathrm{M}$ and SD denote mean and standard deviation, respectively.

A first analysis tested performance for change detection and localization separately. Performance in each task was quantified using the discrimination sensitivity index $\mathrm{d}^{\prime}$, which indicates how reliably signals (here: changes) can be discriminated from noise (Green and Swets, 1966). Detection-d' (referred to as "Det-d" for short) for the two alternative forced choice (AFC) detection task was computed as

Det $-d^{\prime}=\sqrt{2}^{*} z(p H i t)$,

where $z$ denotes the inverse cumulative normal function and $p H i t$ denotes the proportion of correct trials. Localization- $\mathrm{d}^{\prime}$ (referred to as "Loc-d" $\mathrm{d}^{\prime}$ for short) for the N-AFC localization task (see Smith, 1982) was computed as

$$
\begin{aligned}
\text { Loc }-d^{\prime} & =K_{N} * \ln \left\{\frac{(N-1)^{*} p H i t}{1-p H i t}\right\}, \\
\text { with } \quad K_{N} & =.86-.085^{*} \ln (N-1),
\end{aligned}
$$

where $\mathrm{N}$ denotes the number of items in the display $(3,5$, or 7 ) and pHit denotes the proportion of correct trials. This formula gives a precise estimate of $\mathrm{d}^{\prime}$-values in an n-AFC task (for further information see Smith, 1982). Det-d' and Loc$\mathrm{d}^{\prime}$ were tested separately in two $2 \times 3$ repeated measures ANOVAs with the factors "change type" (unique vs. nonunique feature change) and "set size" (3, 5, or 7 items). We hypothesized that change detection, as opposed to change localization, was less set size-dependent, in particular for unique feature changes. In addition, we quantified the set size effect by computing the slopes of regression functions that relate performance to the number of items in the display (Treisman \& Gormican, 1988; Treisman \& Gelade, 1980). Shallow slopes are usually interpreted as indicating efficient search while steep slopes are interpreted as more inefficient search. To this end, $d^{\prime}$ values for each set size, change type, and participant were subjected to a linear regression analysis, and the resulting slopes in each change type were tested 
against zero (indicating perfectly shallow slopes) using twosided $t$ tests. Furthermore, slopes were tested for differences between change types using two-sided $t$ test.

A second analysis focused specifically on change detection without localization $\left(\operatorname{Det}^{+} \mathrm{Loc}^{-}\right)$. To this end, a d value was calculated for detection without localization (referred to as "Sens-d" "for short) using only the proportion of $\mathrm{Det}^{+} \mathrm{Loc}^{-}$relative to the number of $\operatorname{Det}^{+} \mathrm{Loc}^{-}$and Det $^{-}$Loc $^{-}$trials (thus excluding trials with correct localization; see Rensink, 2004). Thus, a significant Sens-d' would indicate whether detection without localization occurred more frequently than change blindness, implying abovechance performance. Sens-d' was analyzed with a $2 \times 3$ repeated measures ANOVA with the factors change type (unique vs. non-unique feature change) and set size (3, 5, or 7 items). Furthermore, slopes for $\operatorname{Det}^{+} \mathrm{Loc}^{-}$trials were analyzed with a linear regression analysis as described above.

While we assume that incorrect localization in fact indicates that participants had no knowledge of the location of a change at all, it is possible that participants only slightly mis-localized the change. Thus, $\operatorname{Det}^{+} \operatorname{Loc}^{-}$trials might not indicate change detection in the complete absence of localization, but only imprecise localization. To test this possibility, we tested whether participants had a tendency to select a location close to the changing object. To this end, the distances of all objects to the changing object (target) were ranked from 1 (closest to change) to $\mathrm{N}-1$, where $\mathrm{N}$ again denotes the number of objects in the display. For each set size, ranked distance, and change type, we computed the proportion of mis-localized trials relative to the number of all trials of one specific set size and condition. Note that an observer without any knowledge of the location of the change would select among $\mathrm{N}$ locations at random. On trials where this random selection was incorrect, the proportion of the selected locations (N-1) should be distributed uniformly, yielding an expected average mis-localization of $1 / \mathrm{N}$. To analyze whether $\mathrm{Det}^{+} \mathrm{Loc}^{-}$was based on imprecise localization rather than a complete failure to localize, the proportion of mis-localization was analyzed with $2 \times(\mathrm{N}-$ 1) repeated measures ANOVA with factors change type, and location for each set size separately. P-values of all ANOVAs were Bonferroni corrected (pBF) for multiple comparisons.

Post hoc tests in all analyses were two-sided $t$ tests and if appropriate, $p$ values were Bonferroni-corrected (pBF) for multiple comparisons. Furthermore, we report the effect sizes (partial eta-square, Cohen's $d, \mathrm{dz}$, and f) of the individual tests.

\section{Results}

Change detection performance $\left(\right.$ Det- $\left.\mathrm{d}^{\prime}\right)$ was better for unique feature changes (change type: $\mathrm{F}(1,23)=19.666$, $\mathrm{p}$ $\left.<.001, \eta_{p}^{2}=.461, \mathrm{f}=.925\right)$ and generally decreased with increasing set size $\left(\mathrm{F}(2,46)=136.621, \mathrm{p}<.001, \eta_{p}^{2}=\right.$ $.856, \mathrm{f}=2.438$ ). As hypothesized, detection of unique feature changes was less set size-dependent than detection of non-unique feature changes (set size $\mathrm{x}$ change type interaction: $\mathrm{F}(2,46)=3.484, \mathrm{p}=.039, \eta_{p}^{2}=.132, \mathrm{f}=.39$; see left panel Fig. 2). This result was corroborated by the finding that slopes (relating Det-d' to set size) were less steep for unique feature changes than for non-unique feature changes (slopes of -.148 and -.201 , respectively; $\mathrm{t}(23)=-2.441$, $\mathrm{p}=.023, \mathrm{SD}=.108, \mathrm{dz}=.491)$. Change localization performance $\left(\right.$ Loc- $\left.\mathrm{d}^{\prime}\right)$ was as well superior for unique feature changes $\left(\mathrm{F}(1,23)=90.377, \mathrm{p}<.001, \eta_{p}^{2}=.797, \mathrm{f}=\right.$

\section{Change detection}

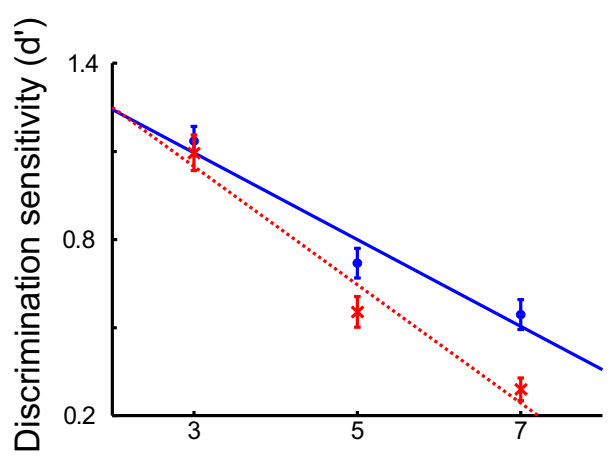

\section{Change localization}

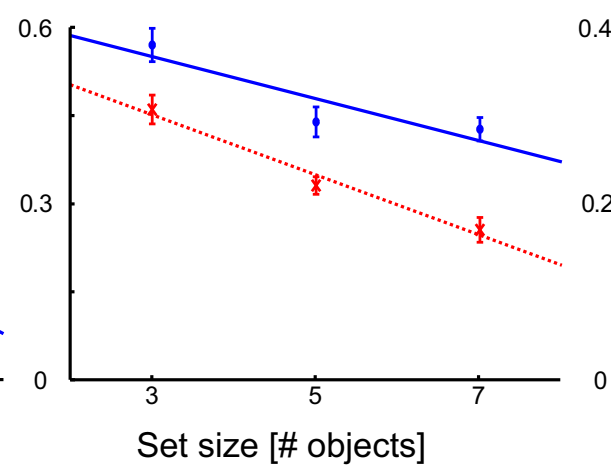

Detection without localization

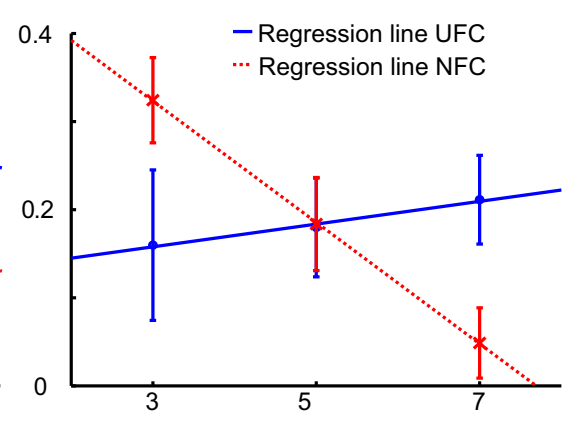

Fig. 2 Dependence of discrimination sensitivity $\left(d^{\prime}\right)$ on set size in Experiment 1. Mean discrimination sensitivity is plotted for each set size, and change detection (left), change localization (middle), and change detection without localization (right). Note that we changed the axis for each plot to improve visibility. Error bars indicate standard errors across participants. Additionally, regression lines are plotted for unique feature changes (UFC, solid blue line) and non-unique feature changes (NFC, dotted red line) 
$1.981)$ and decreased with set size $(F(2,46)=97.775$, $p<$ $\left..001, \eta_{p}^{2}=.810, \mathrm{f}=2.065\right)$. However, in contrast to Det- $\mathrm{d}^{\prime}$, Loc- $\mathrm{d}^{\prime}$ for unique and non-unique feature changes was equally set size-dependent, as indicated by a non-significant set size $\mathrm{x}$ change type interaction $(\mathrm{F}(2,46)=2.68, \mathrm{p}=.079$, $\eta_{p}^{2}=.104, \mathrm{f}=.341$; see middle panel in Fig. 2). The similarity of the set size effect was corroborated by the finding that slopes for Loc-d' were not significantly different between unique and non-unique feature changes (slopes of -.052 and -.037 , respectively; $\mathrm{t}(23)=-1.685, \mathrm{p}=.105$, $\mathrm{SD}=.046, \mathrm{dz}=.343)$. To conform with previous studies, we repeated the analyses using trial proportions as dependent variable (instead of $\mathrm{d}^{\prime}$ ), and were able to replicate all results, including the upcoming analysis of $\mathrm{Det}^{+} \mathrm{Loc}^{-}$trials (see Supplementary material 1). Raw proportions of trials with/without detection and localization can be found in the Supplementary material 2.

Furthermore, we tested whether detection without localization $\left(\operatorname{Det}^{+} \mathrm{Loc}^{-}\right.$) was more prevalent for unique than for non-unique feature changes, and less set size-dependent for unique feature changes (Fig. 2, right panel). Performance on Det $^{+} \mathrm{Loc}^{-}$trials, quantified as Sens-d', was relatively stable across set sizes for unique feature changes, but decreased with set size for non-unique feature changes (set size $\mathrm{x}$ change type interaction: $\mathrm{F}(2,46)=3.899, \mathrm{p}=.027, \eta_{p}^{2}=$ $.145, \mathrm{f}=.412$ ). Separate follow-up ANOVAs confirmed that Sens- $\mathrm{d}^{\prime}$ was set size-independent for unique feature changes $\left(\mathrm{F}(2,46)=.148, \mathrm{pBF}=1, \eta_{p}^{2}=.006, \mathrm{f}=.078\right)$, but strongly set size-dependent for non-unique changes $(\mathrm{F}(2,46)$ $\left.=7.829, \mathrm{pBF}=.002, \eta_{p}^{2}=.254, \mathrm{f}=.57\right)$. Moreover, slopes relating Sens-d' to set size were less steep for unique feature changes than for non-unique feature changes (slopes: .013 and -.069 , respectively; $\mathrm{t}(23)=-2.33, \mathrm{p}=.029, \mathrm{SD}$ $=.172, \mathrm{dz}=.477)$. In fact, slopes were not significantly different from zero for unique feature changes, indicating set size independence $(\mathrm{M}=.013, \mathrm{t}(23)=.518, \mathrm{pBF}=$ $1, \mathrm{SD}=.122, \mathrm{~d}=.107$ ), while a significant slope was present for non-unique feature changes $(\mathrm{M}=-.069, \mathrm{t}(23)$ $=-4.309, \mathrm{pBF}<.001, \mathrm{SD}=.078, \mathrm{~d}=.885)$. For all set sizes considered together, Sens-d $\mathrm{d}^{\prime}$ was similar for unique and non-unique feature changes (change type: $\mathrm{F}(1,23)<$ 1 ), indicating that change detection without localization was not more prevalent for unique feature changes. However, post hoc tests revealed that Sens-d' of unique feature changes was significantly different from chance level in trials with a set size of five objects $(\mathrm{M}=.18, \mathrm{t}(23)=3.137$, $\mathrm{pBF}=.028, \mathrm{SD}=.281, \mathrm{~d}=.641)$ and seven objects $(\mathrm{M}=.211, \mathrm{t}(23)=4.108, \mathrm{pBF}=.003, \mathrm{SD}=.252, \mathrm{~d}=$ $.837)$, but not in trials with three objects $(\mathrm{M}=.16, \mathrm{t}(23)$ $=1.832, \mathrm{pBF}=.48, \mathrm{SD}=.427, \mathrm{~d}=.375)$. For nonunique feature changes we found an opposite trend. Sens- $\mathrm{d}^{\prime}$ of non-unique feature changes was significantly different from chance level in trials with a set size of three objects
$(\mathrm{M}=.324, \mathrm{t}(23)=6.564, \mathrm{pBF}<.001, \mathrm{SD}=.242, \mathrm{~d}=$ $1.134)$ and five objects $(\mathrm{M}=.184, \mathrm{t}(23)=3.404, \mathrm{pBF}=$ $.015, \mathrm{SD}=.264, \mathrm{~d}=.7)$, but not in trials with seven objects $(\mathrm{M}=.049, \mathrm{t}(23)=1.2, \mathrm{pBF}=1, \mathrm{SD}=.2, \mathrm{~d}=.245)$. Significant differences between unique and non-unique change conditions were only found for a set size of seven objects $(\mathrm{M}=.211$ and $\mathrm{M}=.049$, respectively; $\mathrm{t}(23)=-2.687$, $\mathrm{pBF}=.04, \mathrm{SD}=.3, \mathrm{dz}=.543)$, but not for three objects $(\mathrm{M}=.16, \mathrm{M}=.324$, respectively; $\mathrm{t}(23)=.1 .462, \mathrm{pBF}=$ $.472, \mathrm{SD}=.551, \mathrm{dz}=.3)$ and five objects $(\mathrm{M}=.18, \mathrm{M}=$ .184 , respectively; $\mathrm{t}(23)=.0 .059, \mathrm{pBF}=1, \mathrm{SD}=.06, \mathrm{dz}=$ $.067)$.

The above-mentioned analyses demonstrate that observers sometimes detected changes without localizing them correctly. However, it is conceivable that trials labeled as $\operatorname{Det}^{+} \operatorname{Loc}^{-}$are not actually due to a complete failure of localization, but rather due to imprecise localization. Thus, for trials with incorrect localization, we tested whether observers had a tendency to locate the change in the proximity of the correct location. For each set size separately, locations were sorted according to their proximity to the changing object and we analyzed how often participants chose each of the sorted locations using a 2 (change type: unique vs. non-unique changes) $\mathrm{x}$ set size-1 (location) ANOVA. No significant location effects were found for any set size, indicating that when localization was incorrect, participants did not locate the change close to the changing object (Fig. 3), but selected a location at random (set size $3: \mathrm{F}(1,23)=6.117, \mathrm{pBF}=.063, \eta_{p}^{2}=.21, \mathrm{f}=.516$; set size 5: $\mathrm{F}(3,69)=1.766, \mathrm{pBF}=.486, \eta_{p}^{2}=.071, \mathrm{f}=.276$; set size 7: $\left.\mathrm{F}(5,115)=.789, \mathrm{pBF}=1, \eta_{p}^{2}=.033, \mathrm{f}=.185\right)$. Moreover, there was no difference between unique and non-unique changes regarding the choice of locations, as indicated by non-significant change type $\mathrm{x}$ location interactions (set size $3: \mathrm{F}(1,23)=1.403, \mathrm{pBF}=.744, \eta_{p}^{2}=.058$, $\mathrm{f}=.248$; set size $5: \mathrm{F}(3,69)=1.442, \mathrm{pBF}=.714, \eta_{p}^{2}=$ $.059, \mathrm{f}=.25$; set size $7: \mathrm{F}(5,115)=.801, \mathrm{pBF}=1, \eta_{p}^{2}=$ $.034, \mathrm{f}=.188)$.

\section{Discussion}

In this experiment, we tested (1) whether the presence of a change can be "sensed" even without conscious localization of the changing object, and (2) under which conditions this sensing occurs. We hypothesized that observers can detect changes without localizing them only when a unique feature appears or disappears from the display. Moreover, due to the pre-attentive representation of feature values (Quinlan, 2003; Treisman \& Gelade, 1980), detection without localization of unique features should be independent of set size. By contrast, when a non-unique feature changes, observers may only detect and localize the change if their focus of 


\section{Mis-localization Detection without localization}

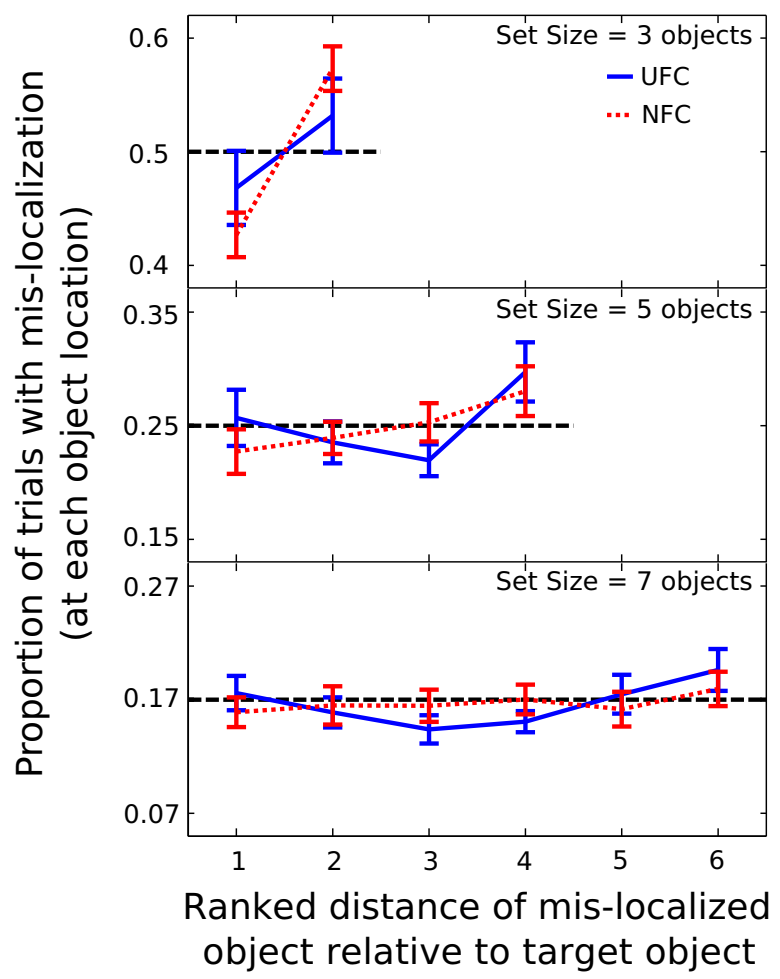

Fig. 3 Mis-localization for change detection without localization in Experiment 1. Incorrect choices in the localization task were ranked according to the distance between the changed object and the incorrectly selected object. Unique feature changes (UFC) are displayed in blue (solid line) and non-unique feature changes (NFC) are displayed in red (dotted line). Error bars indicate standard errors across participants. Horizontal dashed lines indicate the expected uniform mis-localization indicative of random guessing of locations

attention happens to be allocated to the changing object, but will be change blind if attention is allocated elsewhere.

As predicted, detection of unique feature changes was less set size-dependent than detection of non-unique feature changes, indicating that less attentional resources have to be recruited for detecting unique feature changes. By contrast, localization performance was equally set size-dependent for both change types. This finding is consistent with the idea that localization, unlike detection, requires the deployment of spatial attention for both change types (unique vs. non-unique feature changes). Furthermore, we found that participants sometimes detected changes without localizing them. Moreover, we found that the occurrence of change detection without localization for unique feature changes was independent of set size, indicating that "sensing" of unique feature changes can occur independently of the allocation of spatial attention. Importantly, when participants could not report the location of the change, they selected a location at random. Thus, localization was either accurate or failed completely. This implies that trials labeled as "detection without localization" did not comprise trials with partial localization.

While participants were able to sense unique feature changes even in trials with larger set sizes, non-unique feature changes were sensed only in trials with small set sizes. This contradicts our initial hypothesis that changes of non-unique features cannot be sensed at all. However, sensing might not only be based on registering the presence or absence of a unique color but also on registering a change in average luminance across scenes. Importantly, changes in the present study consisted always of a luminance change (dark to a pale color or vice versa) within one hue. Luminance-just like color and orientation-has been proposed to be encoded pre-attentively (Treisman \& Gelade, 1980; Treisman \& Gormican, 1988) and feature maps might have coded the average luminance in each scene (Chong \& Treisman, 2003; Parkes et al., 2001). Several studies have shown that such ensemble statistics of a scene can be established outside the focus of attention and without awareness of the scene's individual elements (Alvarez \& Oliva, 2009). Moreover, changes in ensemble statistics can be detected without awareness of the changing elements (Haberman \& Whitney, 2011; Howe \& Webb, 2014). Thus, detection without localization for non-unique features might have been based on a representation of the scene's ensemble statistics, since at small set sizes, even a non-unique change could bring about a change in the scene's ensemble luminance or hue. However, the impact of a non-unique feature change on the ensemble representation diminishes with increasing set size. This would explain the steep slopes for $\mathrm{Det}^{+} \mathrm{Loc}^{-}$trials for non-unique feature changes. By contrast, when a unique feature changes, Det $^{+} \mathrm{Loc}^{-}$is independent of set size because the change signal always involves the appearance or disappearance of one unique color in addition to the change in ensemble statistics. Hence, only the change of unique features provides a sufficiently strong change signal for sensing at large set sizes.

\section{Experiment 2: Sensing and identification of changes}

Experiment 1 revealed that change detection can occur without localization. But does this finding also imply that participants were not able to identify the change? To conclude that change detection without localization implies also the absence of identification, we conducted a second experiment in which we tested participants' detection, localization, and identification performance with a fixed set size of seven objects. 


\section{Methods}

The procedure of Experiment 2 was identical to the one used in Experiment 1, except for the following changes. An independent sample of 30 participants was tested (mean age: $27.5 \pm 5$ SD; 21 women, nine men; three left-handed) after giving signed informed consent.

The experiment was held in one session of approximately $1 \mathrm{~h}$ and included 170 trials, preceded by $24-48$ training trials. Set size was fixed at 7 items. After the presentation sequence ended, participants responded to three questions: detection, localization, and identification. For identification, all ten colors used in this study (see Methods section of Experiment 1) were displayed as a row of colored bars (see Fig. 1, top). The positions of the colors were randomized. Participants were instructed to select the color of the changing object either from before or after the change by mouse click. Thus, with two possible options out of ten alternatives, the task was equivalent to a $5 \mathrm{AFC}$ task.

\section{Analysis}

The first part of the analysis pipeline was identical to Experiment 1. To replicate the findings, we calculated once more Sens-d' values based on the proportion of $\mathrm{Det}^{+} \mathrm{Loc}^{-}$ relative to the number of $\mathrm{Det}^{+} \mathrm{Loc}^{-}$and $\operatorname{Det}^{-} \mathrm{Loc}^{-}$trials (irrespective of identification performance). Afterwards, we compared Sens-d' between unique feature changes and non-unique feature changes (two-sided $t$ tests) and tested whether Sens-d' was different from chance level in either condition. Additionally, we calculated the mis-localization in Det $^{+} \mathrm{Loc}^{-}$trials (see Experiment 1) and tested for differences using a repeated measures ANOVA with factors change type and location.

In the second part of the analysis, we tested whether participants ever correctly identify the change without localizing it. In other words, does change detection without localization also imply a failure of identification? If so, the proportion of identification under sensing (based on the proportion of $\operatorname{Det}^{+} \mathrm{Loc}^{-} \mathrm{Id}^{+}$relative to the number of $\mathrm{Det}^{+} \mathrm{Loc}^{-} \mathrm{Id}^{+}$and $\mathrm{Det}^{+} \mathrm{Loc}^{-} \mathrm{Id}^{-}$trials) should remain at chance level (i.e., performance of .20; two-sided $t$ test).

\section{Results}

As in Experiment 1, we found that Sens-d' of unique color changes was higher than Sens-d' of non-unique color changes $(M=.299$ and $M=.037$, respectively; $t(29)$ $=-4.833, \mathrm{p}<.001, \mathrm{SD}=.297, \mathrm{dz}=1.276)$. In fact, Sens-d' exceeded chance level only for the unique feature change condition (unique: $\mathrm{t}(29)=8.511, \mathrm{pBF}<.001, \mathrm{SD}$ $=.192, \mathrm{~d}=1.554$; non-unique: $\mathrm{t}(29)=0.942, \mathrm{pBF}=.708$,
$\mathrm{SD}=.216, \mathrm{~d}=.171)$. When change localization failed, participants did not choose a location close to the changing object (location: $\mathrm{F}(5,145)=1.274, \mathrm{p}=.278, \eta_{p}^{2}=.042$, $\mathrm{f}=.207$ ). As in Experiment 1 , the spatial distribution of mis-localization was similar for unique and non-unique feature changes, as indicated by a non-significant change type $\mathrm{x}$ location interaction $\left(\mathrm{F}(5,145)=.3, \mathrm{p}=.912, \eta_{p}^{2}=.01\right.$, $\mathrm{f}=.101)$. Raw proportions of trials with/without detection and localization can be found in the Supplementary material 2.

As expected, identification performance under sensing did not exceed chance level for unique feature changes. In fact, performance was below chance level $(\mathrm{M}=.06, \mathrm{t}(29)$ $=-18.196, \mathrm{pBF}<.001, \mathrm{SD}=.042, \mathrm{~d}=3.326)$. By contrast, identification performance under sensing was above chance for non-unique changes $(\mathrm{M}=.308, \mathrm{t}(29)=5.936$, $\mathrm{pBF}<.001, \mathrm{SD}=.099, \mathrm{~d}=1.084)$. This pattern of performance was due to a bias to report the non-unique color as changing, possibly because these colors were easier to pick up due to their recurrence in the scene.

To confirm this bias statistically, we compared the proportion of $\mathrm{Det}^{+} \mathrm{Loc}^{-}$trials on which participants reported a unique or non-unique color. Participants were more likely to report a non-unique color as changing in both the unique feature change condition $\left(\mathrm{M}_{\text {non-unique }}=.845\right.$ and $\mathrm{M}_{\text {unique }}=$ $.155 ; \mathrm{t}(29)=16.897, \mathrm{pBF}<.001, \mathrm{SD}=.227, \mathrm{dz}=3.084)$ and in the non-unique feature change condition $\left(\mathrm{M}_{\text {non-unique }}\right.$ $=.792$ and $\mathrm{M}_{\text {unique }}=.208 ; \mathrm{t}(29)=15.231, \mathrm{pBF}<.001$, $\mathrm{SD}=.21, \mathrm{dz}=2.781)$. Note that, by definition, non-unique colors never changed in the unique feature change condition, leading to guessing below chance level. By contrast, this guessing strategy artificially improved performance in the non-unique feature change condition (Fig. 4).

\section{Discussion}

Previous studies have demonstrated that in visual search and change detection experiments, identification is dependent on localization (Treisman \& Gelade, 1980; Treisman \& Gormican, 1988; Dukewich \& Klein, 2009; Ghorashi et al., 2010; Busch et al., 2010a; Schneider \& Wascher, 2013; Chan \& Hayward, 2009; Watanabe, 2003; Wolfe et al., 2006; Agostinelli et al., 1986; Becker et al., 2000; Hughes et al., 2012). Thus, Experiment 2 investigated whether change detection without localization also implies a failure to identify the changing feature. As in Experiment 1, significant change detection without localization was found for unique feature changes, but not for non-unique feature changes. Furthermore, when participants detected changes without localization, they did not systematically choose a location in the vicinity of the correct location, confirming that change detection without localization reflected a complete failure of localization rather than only minor mis-localization. Importantly, when participants detected 

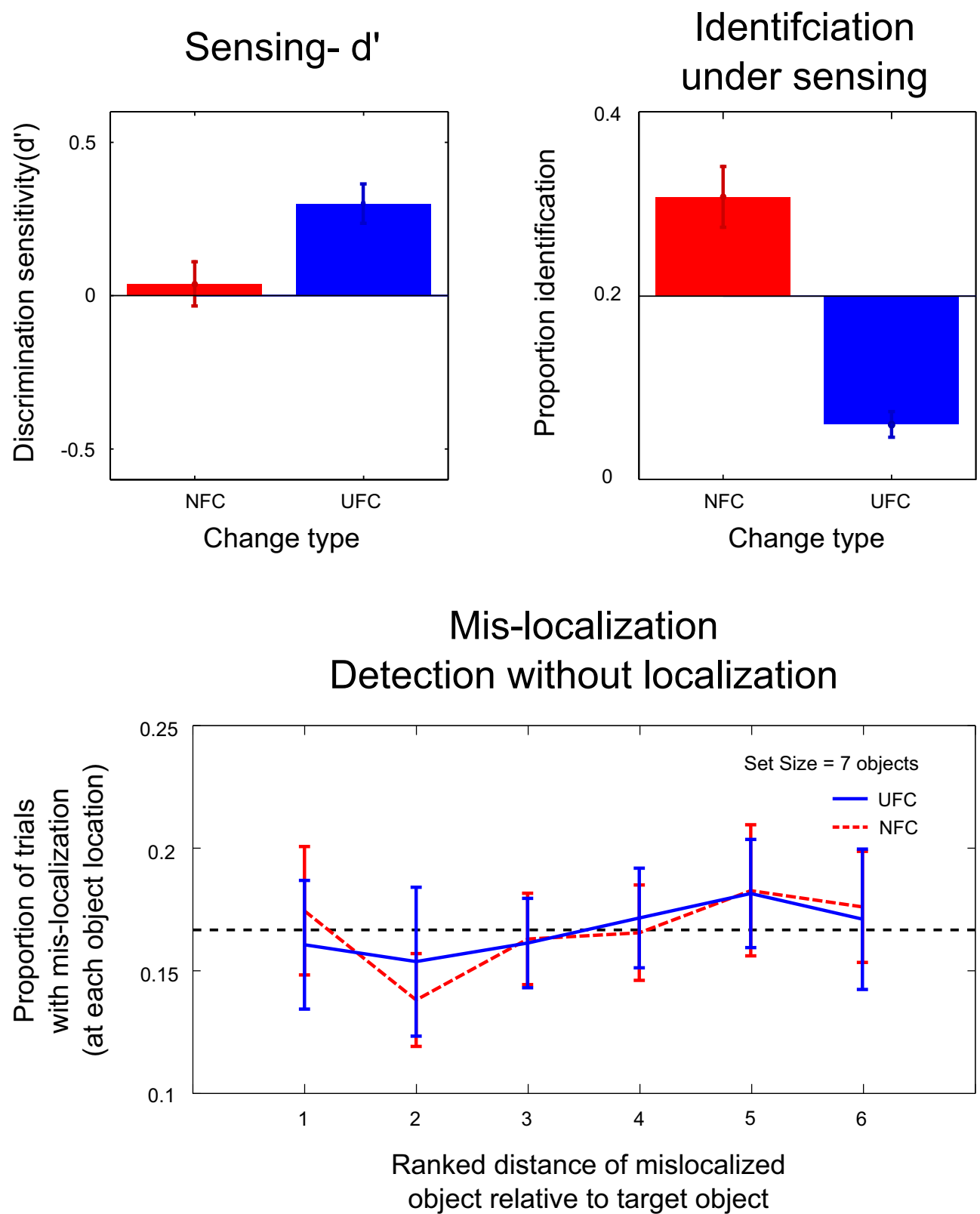

Fig. 4 Summary of performance measures in Experiment 2. The top left graph shows the discrimination sensitivity $\left(\mathrm{d}^{\prime}\right)$ of change detection without localization (sensing) for unique feature changes (UFC) and non-unique feature changes (NFC). Proportion of identification for unique feature and non-unique feature change trials in which participants sensed the change is shown in the top right graph (chance level is 0.2 ). The bottom graph shows mis-localization for change detection

changes without localization, they were not able to identify the changing color-neither the color presented before nor the one presented after the change. Rather, participants were biased to guess that a non-unique color had changed.

These findings indicate that change detection without localization also implies a failure of change identification. without localization. Incorrect choices in the localization task were ranked according to the distance between the changed object and the incorrectly selected object. The horizontal dashed line indicates the expected uniform mis-localization indicative of random guessing of locations. Unique feature changes are displayed in blue (solid line) and non-unique feature changes are displayed in a red dotted line). Error bars indicate standard errors across participants

Therefore, the results allow ruling out that sensing resulted from a post-perceptual and post-attentive inference that a change must have happened, whenever participants noticed a new color. In sum, Experiment 2 confirmed that change detection without localization is due to pre-attentive feature registration. 


\section{Experiments 3a and 3b: The time course of change detection without localization}

Experiments 1 and 2 revealed that change detection can occur without localization and that when localization fails, identification fails as well. But how does change detection without localization evolve over time? Experiments 1 and 2 were not suited for investigating this question since trials were rather short and a change occurred only once in each trial. Thus, Experiments $3 \mathrm{a}$ and $\mathrm{b}$ were designed to study the differential time course of change detection without localization by presenting changes repeatedly in a flicker paradigm.

\section{Methods}

\section{Participants}

We tested 27 participants in Experiment 3a; two participants with less than $10 \%$ correct localization were excluded from the analysis (chance level: $12.5 \%$ ). According to the criteria described below, ten participants were classified as "detecters" (mean age: $23 \pm 2.3 \mathrm{SD}$; seven women, three men; one left-handed) and 15 as "non-detecters" (mean age: $23.2 \pm 2.3 \mathrm{SD}$; all women; all right-handed).

In Experiment 3b, an independent sample of 27 participants was tested. Six participants with less than $10 \%$ correct localization were excluded from the analysis (chance level: $12.5 \%)$. Eleven participants were classified as "detecters" (mean age: $22.1 \pm 2.7 \mathrm{SD}$; ten women, one man; one left-handed) and ten participants as "non-detecters" (mean age: $22.5 \pm 2.3 \mathrm{SD}$; seven women, three men; one lefthanded), using the same criteria for classification as in Experiment 3a.

\section{Stimuli}

Scenes were composed of eight objects with a size of $19 \times 17 \mathrm{~mm}\left(1.55^{\circ}\right.$ visual angle $)$ each. In this experiment, we increased the complexity of the objects to keep the task challenging, because we expected that the repeated presentation of each change would facilitate its detection and localization. Objects were hexagons consisting of three pairs of bars: one pair of black bars, one pair of a unique color and another pair of a non-unique color. Hexagons were placed on two invisible circles at a distance of $2.3^{\circ}$ and $5.3^{\circ}$ from the central fixation cross.

\section{Procedure}

Experiment 3a The experiment was divided into two sessions. Sessions took approximately $1.5 \mathrm{~h}$ each, with presentations of 240 change trials and 60 no-change trials, preceded by 15 training trials. Participants viewed original (A) and changed scenes $\left(\mathrm{A}^{\prime}\right)$ separated by blank screens in sequences of $\mathrm{A}-\mathrm{A}^{\prime}-\mathrm{A}-\mathrm{A}^{\prime} \ldots$. Scenes were presented for $400 \mathrm{~ms}$ and blank displays (white) for $100 \mathrm{~ms}$. Thus, a single cycle consisting of an original and a changed scene lasted one second. A central fixation cross was always visible. Sequences were terminated unpredictably for the participant after $1,3,5,7$, or 9 s. After terminating the presentation sequence, participants answered two questions: "was there a change?" (yes/no) and "where was the change?" (8AFC). The localization question was presented only on change trials. Blank displays were presented during the inter-trial intervals (500-1500 ms; see Fig. 5). Participants were instructed to maintain central fixation during stimulus presentation and to answer as accurately as possible.

Experiment 3b Changes occurred on most trials in Experiment $3 \mathrm{a}$, which might have biased participants to report the presence of a change in the detection task, thus artificially increasing the number of $\mathrm{Det}^{+} \mathrm{Loc}^{-}$responses. Moreover, some participants reported difficulties on trials with a short sequence duration when the preceding trial had a long duration. Thus, Experiment $3 \mathrm{~b}$ was an exact replication of the previous study with only the following three modifications: (1) the experiment used equal numbers of change trials and no-change trials. (2) Only two presentation durations were used (1 and 6 s). (3) Sequence durations were predictable and presented block-wise. Each block was preceded by 12 training trials and consisted of 80 change trials and 80 no-change trials. The order of blocks was counterbalanced across participants.

\section{Analysis}

Studies have demonstrated that change detection without conscious perception of the changing object (sensing) is found only in a subset of trials, and sometimes also only in a subset of participants (Busch et al., 2010b; Howe \& Webb, 2014; Haberman \& Whitney, 2011; Rensink, 2004). Thus, if participants are able to detect changes without localizing them, effects are expected to be rather small. Here, we used a criterion for selecting participants showing sensing based on their proportion of $\mathrm{Det}^{+} \mathrm{Loc}^{-}$(for similar rationale, see Rensink, 2004). Participants were classified as "detecters" if they showed at least $5 \% \operatorname{Det}^{+} \mathrm{Loc}^{-}$responses for each set size and within each change condition. Participants with less than $5 \% \mathrm{Det}^{+} \mathrm{Loc}^{-}$trials were classified as "non-detecters". The rationale for this selection was that we wanted to compare $\operatorname{Det}^{+} \mathrm{Loc}^{-}$between change conditions which requires a sufficient number of trials to analyze in both change conditions. Note that according to the abovementioned criterion, all participants in Experiments 1 and 2 were classified as detecters. 


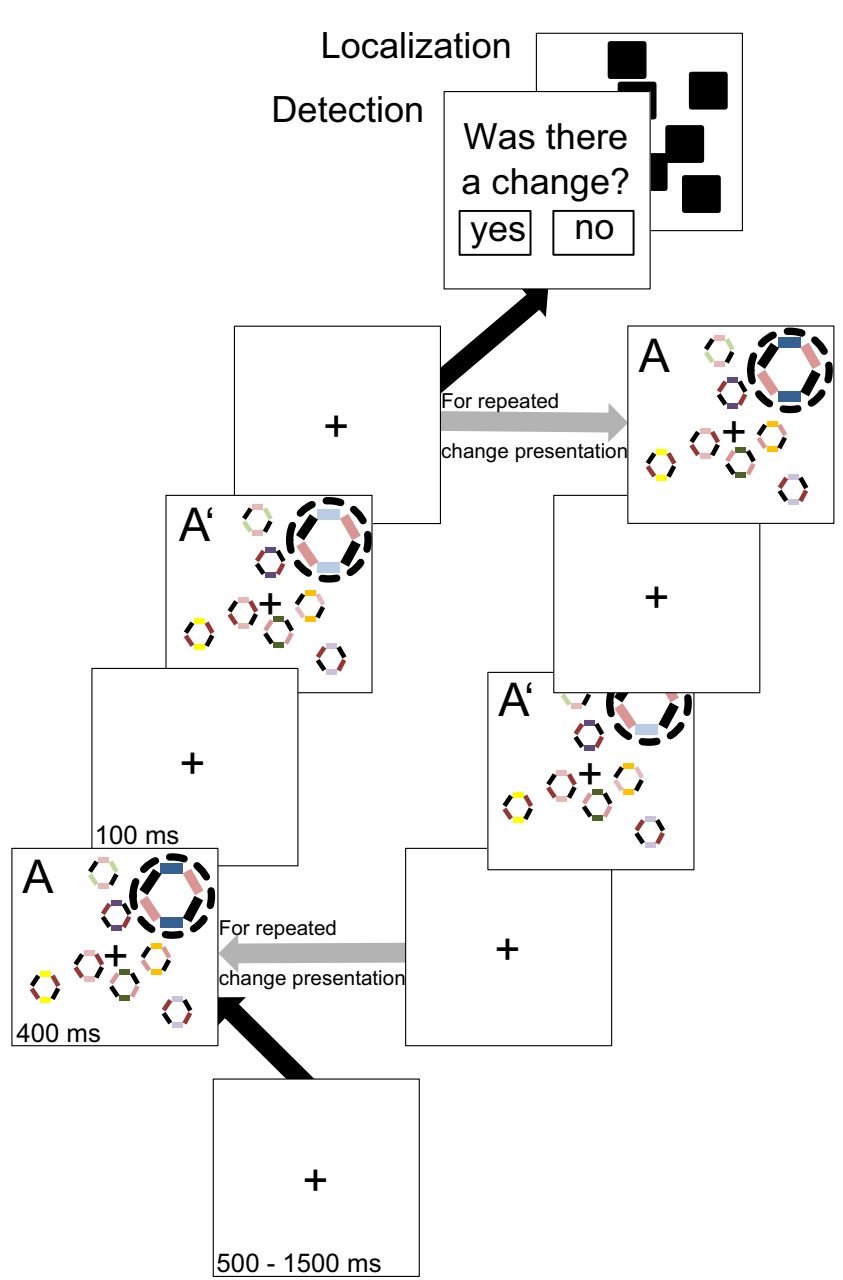

Fig. 5 Experimental Design Experiment 3a and b. Each trial was preceded by a blank display (500-1500 ms). The original scene (A) was substituted by the modified scene $\left(\mathrm{A}^{\prime}\right)$. Scenes were presented for $400 \mathrm{~ms}$ each and interrupted by short blank screens (100 ms). Changes were presented either in a one-shot sequence $\left(\mathrm{A}, \mathrm{A}^{\prime}\right)$ or presented repeatedly for up to $9 \mathrm{~s}\left(\mathrm{~A}, \mathrm{~A}^{\prime}, \mathrm{A}, \mathrm{A}^{\prime}, \ldots\right)$ indicated by the grey arrows. We show only the unique feature change and the target object is magnified and marked with a dotted circle for illustration. Change displays were followed only by a detection task and localization task display

The analysis pipeline was identical for Experiments 3a and $b$. Performance of detecters was quantified by calculating their Sens-d'. Sens-d' was computed as

Sens $-d^{\prime}=z(p H i t)-z(p F A)$,

where $z$ denotes the inverse cumulative normal function, $p$ Hit denotes the proportion of $\operatorname{Det}^{+} \mathrm{Loc}^{-}$trials (relative to all trials with incorrect localization; see Experiment 1), and $p F A$ denotes the false alarms on no-change trials (Green \& Swets, 1966). To study the time course of change detection without localization and its dependence of the type of change, we compared Sens-d' at each presentation duration between unique feature changes and non-unique feature changes (two-tailed $t$ tests). Additionally, we calculated the mis-localization in $\mathrm{Det}^{+} \mathrm{Loc}^{-}$trials (see Experiment 1) and tested for differences using a repeated measures ANOVA with factors change type, and location.

Dividing the sample of participants into detecters (with at least $5 \% \mathrm{Det}^{+} \mathrm{Loc}^{-}$) and non-detecters necessarily results in two groups that differ in the number of $\operatorname{Det}^{+} \mathrm{Loc}^{-}$trials, but it does not determine how the difference between groups comes about. Specifically, we considered three possible differences between detecters and non-detecters. First, it is conceivable that detection without localization is more prevalent in the group of detecters due to a more liberal decision criterion. Thus, compared to the group of nondetecters, such a bias would make detecters more prone to report that a change had occurred, increasing the number of $\operatorname{Det}^{+} \mathrm{Loc}^{-}$on change-trials, but also the number of false alarms on no-change trials. If this were true, the higher prevalence of $\mathrm{Det}^{+} \mathrm{Loc}^{-}$in the group of detecters would simply be the result of lucky guessing. To test for this possibility, we computed response bias $\mathrm{c}$ as

$c=-\frac{z(p H i t)+z(p F A)}{2}$,

where $p H i t$ denotes the proportion of detected changetrials, and $p F A$ denotes the false alarms on no-change trials (Green \& Swets, 1966). Second, detecters-compared to non-detecters-might be more accurate at detecting changes without being more accurate at localizing them. Thus, detecters might be less often change blind, but with no difference in detection with localization. Finally, detecters might be less accurate at localizing changes, but as often change blind as non-detecters. These three scenarios were tested by comparing the groups' response bias $\mathrm{c}$ and their Loc-d' using ANOVAs with between-subject factor group (detecters vs. non-detecters) and within-subject factors change type (unique vs. non-unique feature change) and presentation duration.

\section{Experiment 3a: Results}

In the group of detecters, a difference in Sens-d' between unique and non-unique feature changes emerged with increasing display durations (see top left panel Fig. 6): while Sens- $\mathrm{d}^{\prime}$ increased for unique feature changes, it remained at chance for non-unique feature changes at all display durations. A significant difference between unique and non-unique feature changes was found for a presentation duration (PD) of $7 \mathrm{~s}$ (mean $\mathrm{d}^{\prime}$ of .319 and -.03 , respectively; $\mathrm{t}(9)=-3.97, \mathrm{pBF}=.017, \mathrm{SD}=.278, \mathrm{dz}=1.158)$ only, as indicated by non-significant differences at all remaining presentation duration (PD $1 \mathrm{s:} \mathrm{t}(9)<1$; PD 3 s: $\mathrm{t}(9)<1$; PD $5 \mathrm{~s}: \mathrm{t}(9)<1$; PD 9 s: $\mathrm{t}(9)=-1.974, \mathrm{pBF}=.399)$. Moreover, at $7 \mathrm{~s}$ duration, Sens-d' was also significantly different from chance (indicating more $\mathrm{Det}^{+} \mathrm{Loc}^{-}$than false alarms) 

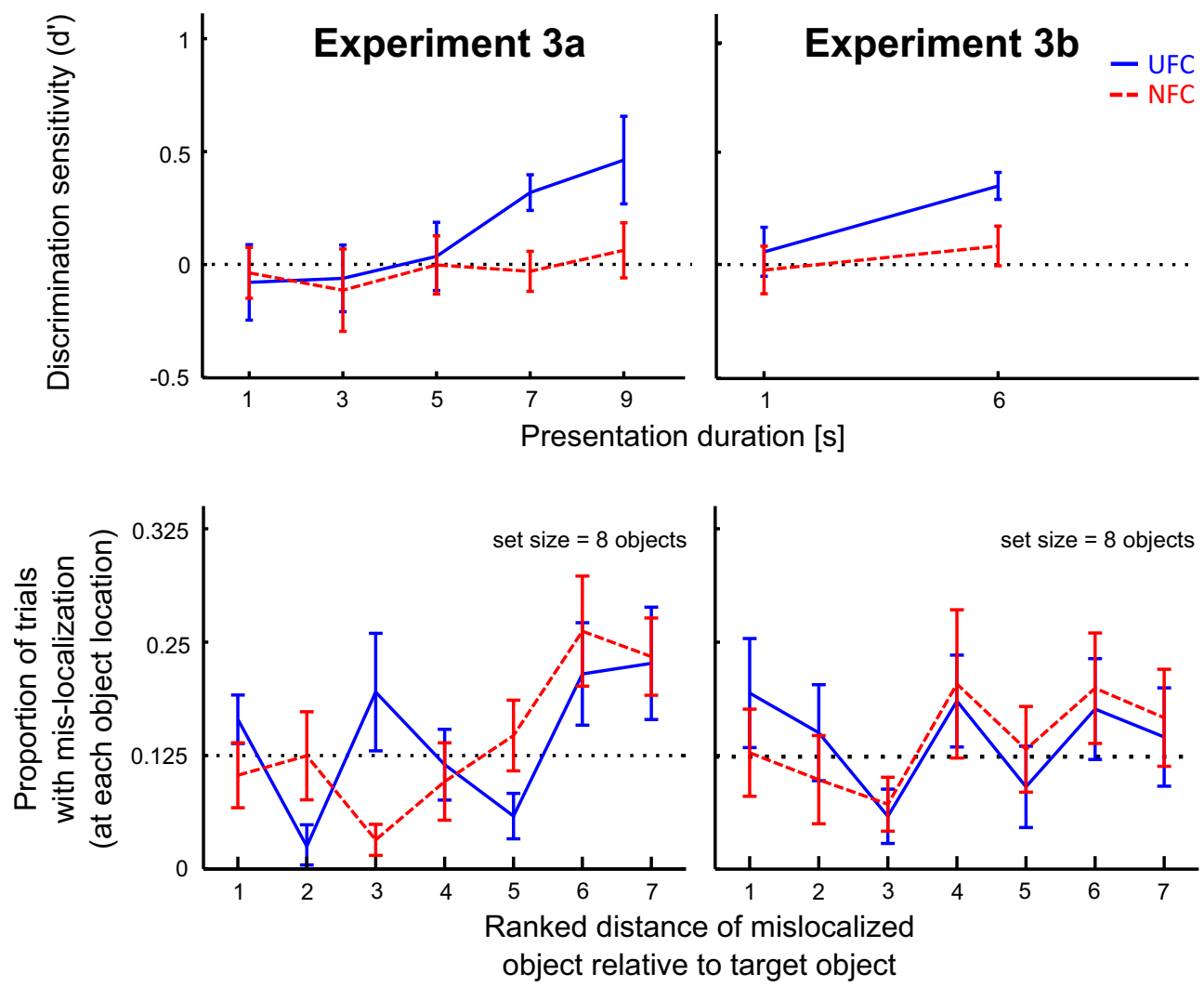

Fig. 6 Discrimination sensitivity $\left(\mathrm{d}^{\prime}\right)$ and mis-localization of detecters for change detection without localization in Experiment $3 \mathrm{a}$ (left) and b (right). Top graphs: Unique feature changes (UFC) in blue (solid line) and non-unique feature changes (NFC) are displayed in red dotted line). The black dashed line indicates chance performance. Bottom graphs: Mis-localization for change detection without localization.

for unique feature changes $(\mathrm{M}=.319, \mathrm{t}(9)=3.826, \mathrm{pBF}=$ $.04, \mathrm{SD}=.263, \mathrm{~d}=.1 .213)$, but not for non-unique feature changes $(\mathrm{M}=-.03, \mathrm{t}(9)=-.321, \mathrm{pBF}=1, \mathrm{SD}=.296, \mathrm{~d}$ $=.101)$. In fact, Sens-d' for non-unique feature changes was insignificant at all presentation durations (all $\mathrm{t}(9)<1$ ). Raw proportions of trials with/without detection and localization can be found in the Supplementary material 2.

The analysis of mis-localization was restricted to the presentation duration of $7 \mathrm{~s}$ (see bottom left panel Fig. 6). When change localization failed, detecters did not tend to chose a location close to the changing object. On the contrary, they tended to chose locations more distant to the changing object, as indicated by a significant location main effect $\left(\mathrm{F}(6,54)=2.968, \mathrm{p}=.014, \eta_{p}^{2}=.248, \mathrm{f}=.574\right)$. As in Experiment 1, the distribution of mis-localization was similar for unique and non-unique feature changes, as indicated by a non-significant change type $\mathrm{x}$ location interaction $\left(\mathrm{F}(6,54)=1.897, \mathrm{p}=.098, \eta_{p}^{2}=.174, \mathrm{f}=.459\right)$.

In the comparison of detecters and non-detecters (see top left panel Fig. 7), we found that the two groups did not use different decision criteria in the detection task, as indi-
Incorrect choices in the localization task were ranked according to the distance between the changed object and the incorrectly selected object. The horizontal dashed line indicates the expected uniform mislocalization indicative of random guessing of locations. Error bars indicate standard errors across participants

cated by a non-significant group main effect $(\mathrm{F}(1,23)=$ 2.857, $\left.\mathrm{p}=.104, \eta_{p}^{2}=.111, \mathrm{f}=.353\right)$ and non-significant interactions (group $\mathrm{x}$ change type: $\mathrm{F}(1,23)=1.109, \mathrm{p}=$ $.303, \eta_{p}^{2}=.046, \mathrm{f}=.22 ;$ group $\mathrm{x}$ presentation duration: $\mathrm{F}(4,92)=.435, \mathrm{p}=.783, \eta_{p}^{2}=.019, \mathrm{f}=.139 ;$ group $\mathrm{x}$ change type $\mathrm{x}$ presentation duration: $\mathrm{F}(4,92)=1.410, \mathrm{p}$ $=.237, \eta_{p}^{2}=.058, \mathrm{f}=.248$ ). In fact, a post hoc twosided $t$ tests revealed that all participants were biased to report the absence of a change (conservative decision criterion), as indicated by a significantly positive response bias value $(\mathrm{M}=.237, \mathrm{t}(24)=2.718, \mathrm{p}=.012, \mathrm{SD}=.436, \mathrm{~d}=$ $.544)$.

However, the analysis of Loc-d $\mathrm{d}^{\prime}$ showed that detecters and non-detecters differed strongly in their ability to correctly localize changes (see bottom left panel Fig. 7). Although non-detecters were not more accurate in general (group: $\left.\mathrm{F}(1,23)=2.708, \mathrm{p}=.113, \eta_{p}^{2}=.105, \mathrm{f}=.343\right)$, their localization performance improved more with increasing presentation duration (group $\mathrm{x}$ presentation duration: $\left.\mathrm{F}(4,92)=5.719, \mathrm{p}<.001, \eta_{p}^{2}=.199, \mathrm{f}=.498\right)$. No other interaction effects were found (all Fs $<1$ ). 

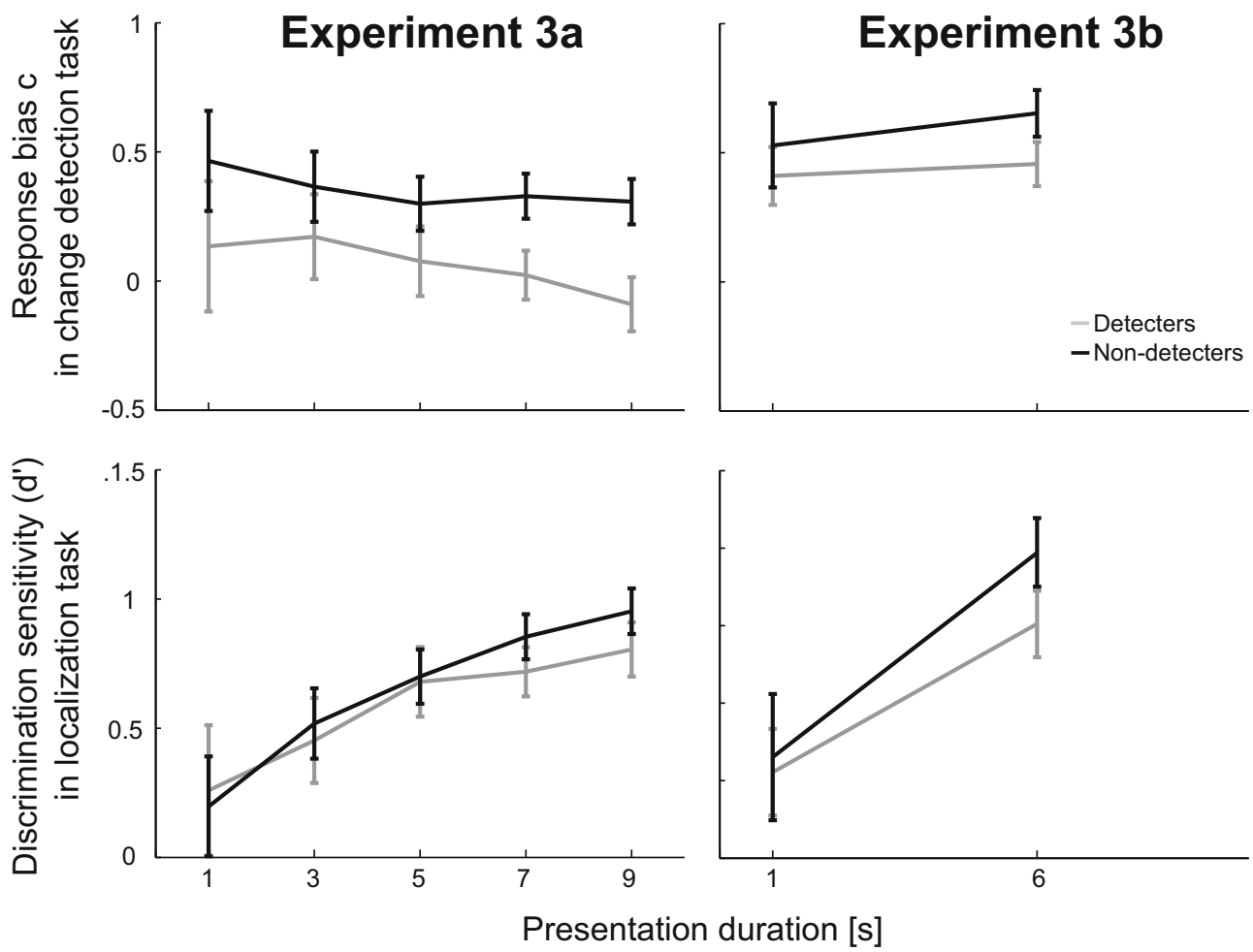

Fig. 7 Difference between detecters (grey) and non-detecters (black) in response bias c (change detection task) and discrimination sensitivity (localization task) in Experiment $3 \mathrm{a}$ (left) and 3b (right). Response bias (top) and localization discrimination sensitivity

\section{Experiment 3b: Results}

In Experiment 3a, we found that significant detection without localization for unique feature changes emerged at longer presentation durations in the group of detecters. However, a statistically significant result (corrected for multiple comparisons) was obtained only for a display duration of $7 \mathrm{~s}$. To confirm that this result was not due to a single false positive, we sought to replicate this effect, focusing only on display durations of $1 \mathrm{~s}$ and $6 \mathrm{~s}$ (see top right panel Fig. 6 for results).

As in Experiment 3a, the detecters' Sens-d' for onesecond-long presentations was at chance $(\mathrm{t}(10)<1)$ and not significantly different for unique and nonunique changes $(\mathrm{t}(10)<1)$. For six-second-long presentations however, Sens-d' was significantly larger for unique than for non-unique feature changes (mean $\mathrm{d}^{\prime}$ of .347 and .081 , respectively; $\mathrm{t}(10)=-4.085, \mathrm{pBF}=$ $.004, \mathrm{SD}=.216, \mathrm{dz}=1.231)$. Moreover, Sens-d $\mathrm{d}^{\prime}$ was above chance level for unique feature changes $(\mathrm{M}=$ $.347, \mathrm{t}(10)=5.539, \mathrm{pBF}<.001, \mathrm{SD}=.208, \mathrm{~d}=$ 1.668) but was at chance level for non-unique changes (bottom) are plotted for all presentation durations. Error bars indicate standard errors across participants. Unique and non-unique feature changes have been combined for illustrative purposes
$(\mathrm{M}=.081, \mathrm{t}(10)=.882, \mathrm{pBF}=1, \mathrm{SD}=.306, \mathrm{~d}$ $=.265)$. Raw proportions of trials with/without detection and localization can be found in the Supplementary material 2.

The analysis of mis-localization in $\operatorname{Det}^{+} \mathrm{Loc}^{-}$trials was restricted to the presentation duration of $6 \mathrm{~s}$ (see bottom right panel Fig. 6). When change localization failed, detecters did not chose a location close to the changing object, but selected a location at random (location: $\mathrm{F}(6,60)$ $<1)$. Moreover, the randomness of localization was similar for unique and non-unique feature changes (change type $\mathrm{x}$ location: $\mathrm{F}(6,60)<1)$.

As in Experiment 3a, detecters and non-detecters did not adopt a different response criterion (see top right panel Fig. 7) as indicated by a non-significant group main effect $\left(\mathrm{F}(1,19)=1.287, \mathrm{p}=.271, \eta_{p}^{2}=.063, \mathrm{f}=.259\right)$ and nonsignificant interactions (all Fs $<1$ ). The decision criterion was significantly positive (i.e., conservative rather than liberal $)$ in all participants $(\mathrm{M}=.508, \mathrm{t}(20)=7.283, \mathrm{p}<.001$, $\mathrm{SD}=.32, \mathrm{~d}=1.588)$. By contrast, non-detecters showed superior Loc-d' (see bottom right panel Fig. 7), as indicated by a significant group main effect $(\mathrm{M}=.524$ and 
$\mathrm{M}=0.413$ Loc- $\mathrm{d}^{\prime}$, respectively; $\mathrm{F}(1,19)=5.147, \mathrm{p}=.035$, $\left.\eta_{p}^{2}=.213, \mathrm{f}=.52\right)$. Other interactions with the factor group were non-significant (all Fs $<2.15$ and $\mathrm{ps}>.159$ ).

\section{Discussion}

Experiment 3 investigated the time course of change detection without localization when changes are repeated for a longer time. The results replicate and extend the findings of the previous experiments. As in Experiments 1 and 2, significant (i.e., more frequent than expected by chance) change detection without localization was found for unique feature changes, but only when the change was repeated for at least 6 (Experiment 3b) or $7 \mathrm{~s}$ (Experiment 3a). By contrast, change detection without localization never occurred for non-unique feature changes regardless of presentation duration.

Unlike in Experiments 1 and 2, not all participants showed change detection without localization, and thus the analysis of $\mathrm{Det}^{+} \mathrm{Loc}^{-}$was restricted to the group of detecters who showed a minimum number of $\operatorname{Det}^{+} \mathrm{Loc}^{-}$trials. A similar finding has been reported by Rensink (2004) and Simons et al. (2005) who found that less than half of their participants reported sensing a change prior to seeing a change. Importantly, detecters and non-detecters in our study were statistically indistinguishable regarding their decision criterion in the detection task. Specifically, detecters did not adopt a significantly more liberal response bias than non-detecters. Rather, we found that the higher proportion of $\operatorname{Det}^{+} \mathrm{Loc}^{-}$trials in detecters resulted from a smaller number of $\mathrm{Det}^{+} \mathrm{Loc}^{+}$trials in this group. Although a subtle difference in criterion might have failed to reach statistical significance due to a lack of statistical power, this finding suggests that detecters differ from non-detecters more in how they process and perceive visual information, rather than in how they decide to report on their perception.

Several authors have suggested that the detecters' higher prevalence of change detection without localization represents a useful skill that serves to increase their vigilance and to prompt a search process, allowing them to respond more efficiently to changes in the environment (Rensink, 2004; Galpin et al., 2008). However, sensing in Experiment 3a and $b$ was only above chance level at longer presentation durations. Prompting a search process at this point is likely to be ineffective since the stimulus presentation is already about to end. Thus, late sensing might help sometimes but not always. Furthermore, one striking difference between detecters and non-detecters was the smaller proportion of trials with successful localization in detecters. Thus, the characteristic performance pattern of detecters appears to be more indicative of a performance deficit, namely, the failure to focus attention to the change region and being able to localize changes.
We can only speculate as to why all observers were labeled as "detecters" in Experiments 1 and 2 and only half of the observers were labeled as "detecters" in Experiment $3 \mathrm{a}$ and $\mathrm{b}$. One reason might be that in Experiments 1 and 2, a change happened on every trial and observers indicated when the change happened in a 2-AFC task. The forced choice relieves observers of the decision as to whether any change has occurred at all, and this relief may be especially important when change localization failed. By contrast, in Experiment 3 observers reported the presence of a change in a yes/no task. Some participants may have found it too odd to report the presence of a change on trials when they did not have a clear perception of where the change happened. Hence, the 2-AFC task might be better suited to identify change detection without localization.

Were the findings in Experiment $3 \mathrm{a}$ and $\mathrm{b}$ a trivial result that was necessitated by the selection procedure? Indeed, by selecting participants with at least $5 \% \mathrm{Det}^{+} \mathrm{Loc}^{-}$trials as detecters, it was trivial to find that detecters showed more detection without localization than non-detecters. However, this study was conducted to investigate under which conditions this behavior occurs and to test the hypothesis that detection without localization specifically occurs when a unique feature changes. By and large, Experiments 1,2 and 3 showed that (A) $d^{\prime}$ for detection without localization was larger for unique than for non-unique feature changes, (B) for unique feature changes, this $\mathrm{d}^{\prime}$ was above chance, and (C) for non-unique feature changes, this $\mathrm{d}^{\prime}$ was at chance. To determine if these results were a trivial consequence of the selection procedure, we conducted a simulation. To mimic the empirical findings, we simulated 27 datasets consisting of a proportion of detection without localization for unique feature changes, for non-unique feature changes, and for false alarms, each ranging randomly from $0-0.1$. As in the real analysis, datasets were selected as "detecters" if performance in both change conditions exceeded $5 \%$. The Matlab code used for the simulation is available as see Supplementary Matlab code. In this simulation, we found a pattern of results as summarized above in only $0.7 \%$ of 30,000 iterations. Thus, the simulation indicates that it is highly unlikely that our findings were a trivial result of the selection procedure.

Due to the long trial duration in a flicker-paradigm, there can be a substantial delay between finding the change and reporting its location. Is it possible that change detection without localization is not due to sensing, but occurs when observers see the change and then forget the location? One argument against this interpretation is that when localization failed, it failed completely, meaning that participants selected items at random and did not tend to select an item close to the change. However, it could be argued that memory traces were completely erased by a "sudden death", as 
described by Zhang and Luck (2009). In their study, three items were presented for $100 \mathrm{~ms}$ and had to be memorized throughout a retention interval of up to $10 \mathrm{~s}$, after which one item had to be reported. They demonstrated that performance declines with longer retention intervals such that memory representations terminated suddenly and completely rather than becoming gradually more imprecise. However, it is unlikely that location information suffered from this kind of sudden death in Experiments $3 a$ and $b$. It is important to note that once a change is found, it is quite obvious to see. Thus, finding the change early on in the trial not only implies a longer delay until the location can be reported, it also implies that the change will be repeated several times over while it is in plain view. Similarly, finding the change essentially reduces the set size down to a single item. Overall, the time to encode the relevant information was longer, the set size was lower, and the delay between flicker offset and report was shorter in Experiment 3 than in the study by Zhang and Luck (2009). Thus, it appears more likely that on trials with correct detection and no localization, participants did not forget the change location, they never saw it in the first place.

\section{General discussion}

In studies on change detection, it is often assumed that being aware of a change allows observers to report on all aspects of the change: its presence, where it occurred, and which object changed. However, observers sometimes report that they had a hunch that something changed without being able to locate or identify the changing object (Busch et al., 2010a; Busch et al., 2010b; Rensink, 2004; Galpin et al., 2008). Here, we investigated under which conditions such change detection without localization occurs.

According to feature integration theory (Treisman \& Gelade, 1980; Treisman \& Gormican, 1988), early vision can be described as a two stage process, wherein visual input is first decomposed into separate feature dimensions such as color or orientation and is represented on feature maps, which code the presence of specific feature values. This stage proceeds rapidly and efficiently in the sense that it works in parallel across the entire scene and does not require focused attention. At the second stage, features (represented on different feature maps) belonging to the same object are bound, forming integrated object representations. This binding process is inefficient in the sense that it requires focused attention and works only on one or few objects at a time. Importantly, while representations at the first stage of unbound features enable detection of feature presence, only integrated object representations allow for object localization and conscious recognition. Numerous studies have investigated whether or not detection, localization, and recognition can be dissociated experimentally in visual search tasks or change detection tasks. Some of these studies found a dissociation of detection, localization, or recognition (Treisman \& Gelade, 1980; Treisman \& Gormican, 1988; Dukewich \& Klein, 2009; Ghorashi et al., 2010; Busch et al., 2010a; Busch et al., 2010b; Schneider \& Wascher, 2013; Chan \& Hayward, 2009; Watanabe, 2003; Wolfe et al., 2006; Agostinelli et al., 1986; Becker et al., 2000; Turatto \& Bridgeman, 2005; Hughes et al., 2012) while others found them to be similar (Luck \& Vogel, 1997; Green, 1992; Joseph et al., 1997; Busey \& Palmer, 2008; Zehetleitner \& Müller, 2010) or found recognition to be conditional upon localization Donk and Meinecke (2001). Thus, whether target detection in visual search for static patterns can occur in the absence of localization or object recognition is still debated.

We hypothesized that change detection without localization or identification occurs when the change happens outside the focus of attention. Such changes would usually go entirely unnoticed, unless the change brings about a modification of one of the feature maps representing the scene. Since feature maps are thought to code only feature presence, such a modification requires the appearance or disappearance of a unique feature that does not belong to other objects in the display (i.e., a feature singleton). Such changes may be registered even outside the focus of attention at a processing stage that precedes feature integration (Haberman \& Whitney, 2011; Alvarez \& Oliva, 2009). However, this registration would allow only to detect that something has changed; it should be insufficient for localization and identification because object recognition requires that features be bound into a coherent or integrated object representation through deployment of focused spatial attention. By contrast, a change of a non-unique or non-singleton feature belonging to multiple objects within the display should be perceivable only under focused attention. Thus, for non-unique feature changes, observers are expected either to detect and localize the change or be completely change blind.

We tested this hypothesis in a series of three change blindness experiments. Changes either involved the change of a unique color (occurring only once in the display) or a non-unique color (present in several objects). Furthermore, we varied the set size in Experiment 1, and changes were either presented once (Experiments 1 and 2) or repeatedly (Experiment $3 \mathrm{a}$ and $\mathrm{b}$ ). In all experiments, we found change detection without localization for unique feature changes. Moreover, detection without localization of unique feature changes was set size independent (Experiment 1) and did not allow for identification of the changed color (Experiment 2). These results indicate that detection (as opposed to localization or identification) of unique feature changes 
is pre-attentive, or at least that the attentional resources required for detection are set size independent. Detection without localization of non-unique changes exceeded chance level only in Experiment 1 and only for small set sizes. Sensing of non-unique feature changes was unexpected, but is likely mediated by detection of a global change in scenes' luminance (see Discussion Experiment 1). Notably, change detection without localization was relatively rare compared to detection with localization and change blindness. As a result, $\mathrm{d}^{\prime}$ for detection without localization was small, albeit significantly above chance and consistent across two experiments. The rareness of detection without localization found in this study and in earlier studies (Busch et al., 2010b; Galpin et al., 2008; Rensink, 2004; Simons et al., 2005; Howe \& Webb, 2014; Haberman \& Whitney, 2011) indicates that mere feature detection is not always available or that observers do not always make use of this information.

Note that the paradigms in the present study differ in important aspects from visual search paradigms that have been frequently employed to study feature integration (Treisman \& Gelade, 1980; Quinlan, 2003; Wolfe, 2003). Visual search tasks are typically used to test the speed of judgments about the presence or absence of a predefined target stimulus. By contrast, the change detection task used in the present study required participants to find changes. Unlike target detection, change detection cannot be performed based on a template of a target stimulus to guide the search process; any object could be the changing object. Moreover, change detection requires more than the encoding of a single scene; observers need to encode the original scene, retain that representation until presentation of the second scene, encode the second scene, then compare both representations and search for a difference (see Rensink, 2002). Therefore, the dependent variable in the present study was not response times as in visual search experiments, but accuracy. Furthermore, even if the process of scene encoding on feature maps was pre-attentive (i.e., not requiring spatial attention), change detection performance-in contrast to visual search response time-is not expected to be strictly independent of set size (Experiment 1) or to occur after the very first presentation of the change (Experiment $3 \mathrm{a}$ and $\mathrm{b}$ ). Thus, a reasonable prediction is that if unique feature changes can be detected pre-attentively without localization, their accuracy vs. set-size functions should be less steep than for non-unique changes and that detection without localization occurs more often for unique that for non-unique changes. Both predictions were indeed confirmed by our results.

We observed that on a fraction of trials, observers correctly reported the location of the change even though they did not detect the change correctly. However, this type of behavior did not occur more often than expected by chance (see Supplement 3). Fernandez-Duque and Thornton (2000) demonstrated that when observers did not detect a change and were then forced to guess between two potential locations, they were more likely to select the correct location where the change had occurred. They interpreted this finding as an effect of implicit or unconscious change localization (but see Mitroff et al., 2002 and Fernandez-Duque and Thornton, 2003 for a debate on the validity of this interpretation). In contrast to these studies, our study was designed for the purpose of testing if observers can sense the occurrence of a change even without conscious change localization, rather than for testing unconscious localization. Thus, participants in our study were to choose a location from among all possible locations in the scene rather than to choose from only two locations as in Fernandez-Duque \& Thornton (2000). Our procedure may not be sensitive enough to reveal effects of unconscious localization. Also, note that sensing that a change occurred is different from implicit change detection since observers are aware of their sensing and report on their awareness in a direct detection task (see also Rensink, 2004). By contrast, implicit change detection has been studied by demonstrating an effect of the presence of a change on performance even when observers do not report the change in a direct detection task (Fernandez-Duque et al., 2003; Fernandez-Duque \& Thornton, 2000; Thornton \& Fernandez-Duque, 2000; Laloyaux et al., 2006; Kimura et al., 2008).

Change detection without localization or identification of the changing object bears resemblance to the phenomenon of "sensing" described by Rensink (2004). "Sensing" refers to the feeling that "something is changing" without a visual experience of the changing object. Rensink studied "sensing" and "seeing" in a change detection task, in which participants were instructed to press one response key when they sensed a change and another key once they saw the change (i.e., they had a visual experience of the object and could verbally localize and describe it). Some participants gave "sense" responses several scene alternations prior to their "see" responses. Importantly, the onset times of sensing and seeing were uncorrelated. Moreover, the average time for seeing relative to sensing in trials when it occurred was the same as the average time for seeing when sensing did not occur. Rensink argued that this pattern of response times suggests that sensing and seeing do not simply correspond to different confidence thresholds, but instead are based on different perceptual mechanisms. However, this interpretation has been vigorously rejected by Simons et al. (2005), who offered a more parsimonious explanation for the temporal lag between "sense" and "see" responses. They showed that the earlier onset of "sensing"-reports compared to "seeing"-reports may be due to a bias to simply guess the presence of a change even before processing any information about the change 
(liberal response bias). Since a change happened on the majority of all trials, this would have been a viable strategy. Moreover, they argued that participants may simply take time to verify their initial detection of the change and withhold their "see" response until they feel confident enough. This verification of the change would cause a similar lag between "sense" and "see" reports. However, this criticism does not explain the differential response to colored flashes (e.g., greatly increased onset of sensing of color changes) shown by Rensink (2004), and it does not apply to the present study in which bias did not contribute to the findings. In Experiments 1 and 2, we used a one-shot presentation and a two-alternative forced choice task, in which observers were forced to choose the presentation interval that was more likely to contain the change. The one-shot presentation does not allow for verification of an initial detection of the change because the change is only presented once. Furthermore, the two-alternative forced choice task rules out a bias to report the presence of a change and it rules out that observers set different confidence thresholds for when to report detection and localization. Thus, the concerns raised by Simons et al. (2005) about the interpretation of sensing changes without seeing do not apply to the findings of change detection without localization in the present study.

Sensing has been studied in a variety of experimental paradigms. For example, Galpin et al. (2008) studied sensing with eye-tracking in a comparative visual search paradigm, where participants were free to report the moment when they started to sense the change and the moment when they detected and localized the change. They showed that participants started to inspect the change region longer before they gave a "sense" response (compared to "see" responses), and that they were more likely to give a "sense" response when their gaze was located within the change region. The authors concluded that "sensing" may arise when a mismatch (e.g., a featural mismatch) is detected within the change region, and when the spatial binding of information within this change region either does not take place or is not maintained. In this study, participants' ability to localize the change was not directly tested. Thus, it is possible that sensing was either "change detection without localization and identification" (as proposed by Rensink, 2004) or "change detection with localization, but without identification". Furthermore, all studies that allowed for a repeated change exposure (Galpin et al., 2008; Rensink, 2004) before giving a sensing response found that sensing was reported only after several seconds (i.e., after repeated change presentation) and only in a subset of participants. This is in line with findings of Experiment $3 \mathrm{a}$ and $\mathrm{b}$, showing that sensing is only sensitive at longer presentation durations. In contrast, one-shot paradigms, in which the change is only presented once, have found change detection without localization or identification consistently in all participants, and the frequency of these responses was greater than expected by chance (Busch et al., 2010b; Howe and Webb, 2014; Haberman \& Whitney, 2011, and the present study). Thus, one-shot paradigms may be particularly sensitive for the sensing phenomenon.

Feature integration theory is closely related to coherence theory (Rensink, 2000; 2002), which holds that most parts of the scene in view are only represented on a pre-attentive level. At this level, the scene is represented by proto-objects (low-level structures which can be fairly detailed). In contrast to feature integration theory, coherence theory assumes that these proto-objects are represented on a spatial map, such that any new information occurring at the same retinal position will overwrite the old proto-object unless attention is focused on the object. Focused attention is needed to make proto-objects coherent (stable and protected against overwriting) in time and space. In contrast to feature integration theory, this coherence is maintained only for as long as attention is focused on the object, and is immediately resolved when attention is withdrawn. Coherence theory explains why focused attention is necessary to see changes. However, it is less clear how coherence theory can accommodate the phenomenon of sensing. Specifically, coherence theory holds that the pre-attentive level of object representation includes some information about the objects' retinal locations. If sensing involves access to this pre-attentive level, sensing should allow observers to localize the change as well as detecting it. It is conceivable, however, that the feeling of sensing is related to the nonattentional "setting" system, which is assumed to guide attention based on pre-attentive processing of scene gist or layout (Rensink, 2002).

The distinction between change detection with and without localization (or identification) is closely related to the distinction between "state-based" and "strength-based" perception, respectively. According to Aly and Yonelinas (2012), "state-based" perception is a discrete perceptual state that does either occur or not occur, and that is accompanied by conscious perception of details of the changing object. By contrast, "strength-based" change detection can vary from weak to strong and is accompanied by a feeling of knowing that something has changed, but with little to no ability to identify what the change was. Using receiver operating characteristics, Aly and Yonelinas (2012) demonstrated that both types of perceptual judgments contribute independently to change detection performance. In later studies, the authors demonstrated that hippocampal damage impairs strength- but not state-based scene perception, and that hippocampal activation measured in fMRI correlates with strength-based, but not state-based perception (Aly et al., 2013a). By contrast, state-based perception was specifically related to activity in regions in the lateral and 
medial posterior parietal cortex (Aly et al., 2013b). Thus, these studies add to the literature suggesting the existence of functionally and phenomenologically different change detection mechanisms (Rensink, 2004; Galpin et al., 2008). Moreover, they also support previous reports of distinct neural signatures for change detection with and without localization/identification (Busch et al., 2010a; Busch et al., 2010b). In light of the present findings, we propose that strength-based change detection relates to pre-attentive registration of stimulus features on maps of unbound features, while state-based change detection relates to the perception of integrated objects following a capacity-limited and attention-demanding integration process.

\section{Conclusions}

In this study, we investigated under which conditions the detection of visual changes can proceed without localization and identification. Based on feature integration theory, we hypothesized that when a change happens outside the focus of attention, such changes are still detectable even without feature-integration if the change brings about a modification of one of the feature maps representing the scene. Only changes of unique colors were detected without localization, and this sensing was independent of set size, confirming that detection can occur without the focused attention that is necessary for feature integration. Whether changes of other pre-attentive features such as orientation, shape and motion can give rise to this kind of perception remains subject to future studies. In sum, our findings suggest that pre-attentive registration of a change on a feature map can support a particular kind of conscious experience even when feature binding has failed: that something has changed somewhere without knowing what or where.

Notes This work was supported by the German Research Foundation (Grant \# BU2400/1-1). We would like to thank Michał Klincewicz and Jacob Shier for their comments on our manuscript, and we would like to thank Anne Elzemann, Svea C. Schröder, and Monika Graumann for help with stimulus collection and data acquisition.

\section{References}

Agostinelli, G., Sherman, S. J., Fazio, R. H., \& Hearst, E. S. (1986). Detecting and identifying change: Additions versus deletions. Journal of Experimental Psychology. Human Perception and Performance, 12(4), 445-454.

Alvarez, G. A., \& Oliva, A. (2009). Spatial ensemble statistics are efficient codes that can be represented with reduced attention. PNAS, 106(18), 7345-7350.
Aly, M., Ranganath, C., \& Yonelinas, A. P. (2013a). Detecting changes in scenes: The hippocampus is critical for strength-based perception. Neuron, 78(6), 1127-1137.

Aly, M., Ranganath, C., \& Yonelinas, A. P. (2013b). Neural correlates of state- and strength-based perception. Journal of Cognitive Neuroscience. accepted.

Aly, M., \& Yonelinas, A. P. (2012). Bridging consciousness and cognition in memory and perception: Evidence for both state and strength processes. PLoS One, 7(1), e30231.

Ball, F., Elzemann, A., \& Busch, N. A. (2013). The scene and the unseen: Manipulating photographs for experiments on change blindness and scene memory. Behavior Research Methods. Advance online publication.

Becker, M. W., Pashler, H., \& Anstis, S. M. (2000). The role of iconic memory in change-detection tasks. Perception, 29(3), 273-286.

Brainard, D. H. (1997). The psychophysics toolbox. Spatial Vision, 10(4), 433-436.

Busch, N. A., Dürschmid, S., \& Herrmann, C. S. (2010a). ERP effects of change localization, change identification and change blindness. Neuroreport, 21(5), 371-375.

Busch, N. A., Fründ, I., \& Herrmann, C. S. (2010b). Electrophysiological evidence for different types of change detection and change blindness. Journal of Cognitive Neuroscience, 22(8), 1852-1869.

Busey, T., \& Palmer, J. (2008). Set-size effects for identification versus localization depend on the visual search task. Journal of Experimental Psychology. Human Perception and Performance, 34(4), $790-810$.

Chan, L. K., \& Hayward, W. G. (2009). Feature integration theory revisited: dissociating feature detection and attentional guidance in visual search. Journal of Experimental Psychology. Human Perception and Performance, 35(1), 119.

Chong, S. C., \& Treisman, A. (2003). Representation of statistical properties. Vision Research, 43(4), 393-404.

Donk, M., \& Meinecke, C. (2001). Feature localization and identification. Acta Psychologica, 106(1-2), 97-119.

Dukewich, K. R., \& Klein, R. M. (2009). Finding the target in search tasks using detection, localization, and identification responses. Canadian Journal of Experimental Psychology = Revue canadienne de psychologie expérimentale, 63(1), $1-7$.

Fernandez-Duque, D., Grossi, G., Thornton, I. M., \& Neville, H. .J. (2003). Representation of change: Separate electrophysiological markers of attention, awareness, and implicit processing. Journal of Cognitive Neuroscience, 15(4), 491-507.

Fernandez-Duque, D., \& Thornton, I. M. (2000). Change detection without awareness: Do explicit reports underestimate the representation of change in the visual system Visual Cognition, 7(1-3), 323-344.

Fernandez-Duque, D., \& Thornton, I. M. (2003). Explicit mechanisms do not account for implicit localization and identification of change: An empirical reply to Mitroff et al. (2002). J Exp Psychol Hum Percept Perform, 29(5), 846-858.

Galpin, A., Underwood, G., \& Chapman, P. (2008). Sensing without seeing in comparative visual search. Consciousness and Cognition, 17(3), 672-687.

Ghorashi, S., Enns, J. T., Klein, R. M., \& Di Lollo, V. (2010). Spatial selection and target identification are separable processes in visual search. Journal of Vision, 10(3), 1-12.

Green, D. M., \& Swets, J. A. (1966). Signal detection theory and psychophysics. New York: Wiley.

Green, M. (1992). Visual search: Detection, identification, and localization. Perception, 21(6), 765-777.

Haberman, J., \& Whitney, D. (2011). Efficient summary statistical representation when change localization fails. Psychonomic Bulletin \& Review, 18(5), 855-859. 
Hayhoe, M. M., Bensinger, D. G., \& Ballard, D. H. (1998). Task constraints in visual working memory. Vision Research, 38(1), 125137.

Howe, P. D. L., \& Webb, M. E. (2014). Detecting unidentified changes. PLOS ONE, 9(1).

Hughes, H. C., Caplovitz, G. P., Loucks, R. A., \& Fendrich, R. (2012). Attentive and pre-attentive processes in change detection and identification. PLoS One, 7(8), e42851.

Joseph, J. S., Chun, M. M., \& Nakayama, K. (1997). Attentional requirements in a 'preattentive' feature search task. Nature, 387(6635), 805-807.

Kimura, M., Katayama, J., \& Ohira, H. (2008). Event-related brain potential evidence for implicit change detection: A replication of Fernandez-Duque et al. (2003). Neuroscience Letters, 448(3), 236-239.

Laloyaux, C., Destrebecqz, A., \& Cleeremans, A. (2006). Implicit change identification: A replication of Fernandez-Duque and Thornton (2003). Journal of Experimental Psychology. Human Perception and Performance, 32(6), 1366-1379.

Luck, S. J., \& Vogel, E. K. (1997). The capacity of visual working memory for features and conjunctions. Nature, 390(6657), 279281.

McConkie, G. W., \& Currie, C. B. (1996). Visual stability across saccades while viewing complex pictures. Journal of Experimental Psychology. Human Perception and Performance, 22(3), 563581.

Mitroff, S. R., Simons, D. J., \& Franconeri, S. L. (2002). The siren song of implicit change detection. J Exp Psychol Hum Percept Perform, 28(4), 798-815.

O'Regan, J. K., Deubel, H., Clark, J. J., \& Rensink, R. A. (2000). Picture changes during blinks: Looking without seeing and seeing without looking. Visual Cognition, 7, 191-211.

O’Regan, J. K., Rensink, R. A., \& Clark, J. J. (1999). Changeblindness as a result of 'mudsplashes'. Nature, 398(6722), 34.

Parkes, L., Lund, J., Angelucci, A., Solomon, J., \& Morgan, M. (2001). Compulsory averaging of crowded orientation signals in human vision. Nature Neuroscience, 4(2001), 739-744.

Quinlan, P. T. (2003). Visual feature integration theory: Past, present, and future. Psychological Bulletin, 129(5), 643-673.

Rensink, R. A. (2000). The dynamic representation of scenes. Visual Cognition, 7, 17-42.
Rensink, R. A. (2002). Change detection. Annual Review of Psychology, 53, 245-277.

Rensink, R. A. (2004). Visual sensing without seeing. Psychological Science, 15(1), 27-32.

Rensink, R. A., O'Regan, J. K., \& Clark, J. J. (1997). To see or not to see: The need for attention to perceive changes in scenes. Psychological Science, 8(5), 368-373.

Schneider, D., \& Wascher, E. (2013). Mechanisms of target localization in visual change detection: An interplay of gating and filtering. Behavioural Brain Research, 256(0), 311-319.

Simons, D. J., Nevarez, G., \& Boot, W. R. (2005). Visual sensing IS seeing: Why 'mindsight', in hindsight, is blind. Psychological Science, 16(7), 520-524.

Simons, D. J., \& Rensink, R. A. (2005). Change blindness: Past, present, and future. Trends in Cognitive Sciences, 9(1), 16-20.

Smith, J. (1982). Simple algorithms for m-alternative forced-choice calculations. Perception \& Psychophysics, 31(1), 95-96.

Thornton, I. M., \& Fernandez-Duque, D. (2000). An implicit measure of undetected change. Spatial Vision, 14(1), 21-44.

Treisman, A., \& Gelade, G. (1980). A feature-integration theory of attention. Cognitive Psychology, 12(1), 97-136.

Treisman, A., \& Gormican, S. (1988). Feature analysis in early vision: Evidence from search asymmetries. Psychological Review, 95(1), $15-48$.

Turatto, M., \& Bridgeman, B. (2005). Change perception using visual transients: Object substitution and deletion. Experimental Brain Research, 167(4), 595-608.

Watanabe, K. (2003). Differential effect of distractor timing on localizing versus identifying visual changes. Cognition, 88(2), 243257.

Wolfe, J. M. (2003). Moving towards solutions to some enduring controversies in visual search. Trends in Cognitive Sciences, 7(2), 7076.

Wolfe, J. M., Reinecke, A., \& Brawn, P. (2006). Why don't we see changes? The role of attentional bottlenecks and limited visual memory. Visual Cognition, 14(4-8), 749-780.

Zehetleitner, M., \& Müller, H. J. (2010). Salience from the decision perspective: You know where it is before you know it is there. Journal of Vision, 10(14).

Zhang, W., \& Luck, S. J. (2009). Sudden death and gradual decay in visual working memory. Psychological Science, 20(4), 423-428. 